\section{BWXT}

Protecting America's Future

Y-12

NATIONAL

SECURITY

COMPLEX

\section{Y-12 GROUNDWATER PROTECTION PROGRAM GROUNDWATER AND SURFACE WATER SAMPLING AND ANALYSIS PLAN FOR CALENDAR YEAR 2008}

\author{
September 2007
}

Prepared by

ELVADO ENVIRONMENTAL LLC Under Subcontract No. 4300054638

for the

Environmental Compliance Department Environment, Safety, and Health Division Y-12 National Security Complex Oak Ridge, Tennessee 37831

Managed by

BWXT Y-12, L.L.C.

for the U.S. DEPARTMENT OF ENERGY under contract No. DE-AC05-00OR22800 


\section{DISCLAIMER}

This report was prepared as an account of work sponsored by an agency of the United States Government. Neither the United States Government nor any agency thereof, nor any of their employees, makes any warranty, express or implied, or assumes any legal liability or responsibility for the accuracy, completeness, or usefulness of any information, apparatus, product, or process disclosed, or represents that its use would not infringe privately owned rights. Reference herein to any specific commercial product, process, or service by trade name, trademark, manufacturer, or otherwise, does not necessarily constitute or imply its endorsement, recommendation, or favoring of the United States Government or any agency thereof. The views and opinions of authors expressed herein do not necessarily state or reflect those of the United States Government or any agency thereof. 


\title{
Y-12 GROUNDWATER PROTECTION PROGRAM GROUNDWATER AND SURFACE WATER SAMPLING AND ANALYSIS PLAN \\ FOR CALENDAR YEAR 2008
}

September 2007

Prepared by

ELVADO ENVIRONMENTAL LLC

Under Subcontract No. 4300054638

for the

Environmental Compliance Department Environment, Safety, and Health Division Y-12 National Security Complex

Oak Ridge, Tennessee 37831

\author{
Managed by \\ BWXT Y-12, L.L.C. \\ for the U.S. DEPARTMENT OF ENERGY \\ under contract No. DE-AC05-00OR22800
}




\section{TABLE OF CONTENTS}

Section $\quad \underline{\text { Page }}$

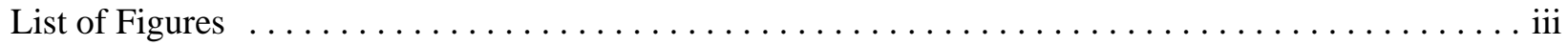

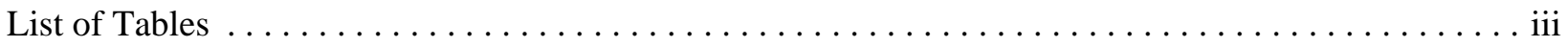

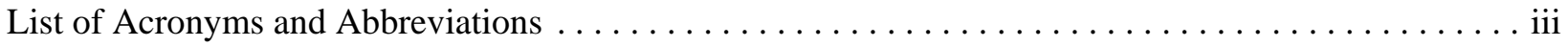

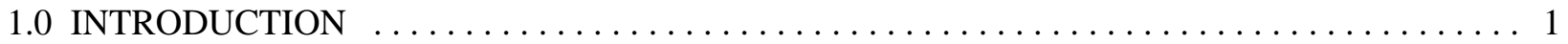

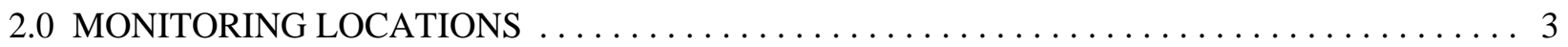

3.0 FIELD MEASUREMENTS AND ANALYTICAL PARAMETERS $\ldots \ldots \ldots \ldots \ldots \ldots \ldots$

4.0 SAMPLE PLANNING, COLLECTION, AND HANDLING $\ldots \ldots \ldots \ldots \ldots \ldots \ldots \ldots \ldots .7$

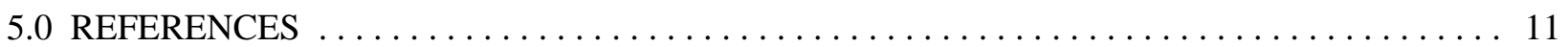

\section{APPENDICES:}

A FIGURES

B TABLES

C CY 2008 GROUNDWATER MONITORING SCHEDULES

D ADDENDA TO THE CY 2008 SAMPLING AND ANALYSIS PLAN

E LABORATORY REQUIREMENTS

(Bottle Lists, Holding Times, Turnaround Times, Elevated Minimum Detectable Activity)

F CY 2008 SAMPLING FREQUENCY FOR MONITORING WELLS

G MANAGEMENT OF PURGED GROUNDWATER 


\section{List of Figures}

Figure

Page

A.1 Hydrogeologic regimes at the Y-12 National Security Complex $\ldots \ldots \ldots \ldots \ldots \ldots \ldots$ A-1

A.2 CY 2008 sampling locations in the Bear Creek Hydrogeologic Regime . . . . . . . . . . A-2

A.3 CY 2008 sampling locations in the Chestnut Ridge Hydrogeologic Regime ........... A-3

A.4 CY 2008 sampling locations in the Upper East Fork Poplar Creek

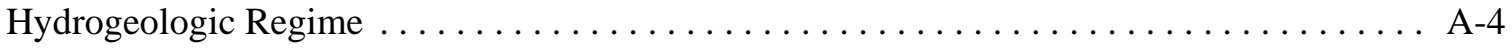

A.5 CY 2008 surface water sampling locations north of Pine Ridge $\ldots \ldots \ldots \ldots \ldots \ldots \ldots$ A-5

A.6 Westbay ${ }^{\mathrm{TM}}$ monitoring system sampling port depths in well GW-722 . . . . . . . . A -6

\section{List of Tables}

$\underline{\text { Table }}$

Page

B.1 Sampling locations, frequency, and analytical parameters for groundwater and surface water monitoring during CY $2008 \ldots \ldots \ldots \ldots \ldots \ldots \ldots \ldots \ldots \ldots \ldots \ldots \ldots \ldots \ldots$

B.2 Field measurements and analytes that comprise the elementary parameter groups for CY 2008 groundwater and surface water samples

\section{List of Acronyms and Abbreviations}

ACO

Bear Creek Regime

BWXT

Chestnut Ridge Regime

CY

DOE

East Fork Regime

EPA

GWPP

GWMS

MAROS

PDB

REDOX

VOCs

$\mathrm{Y}-12$

\author{
Analytical Chemistry Organization \\ Bear Creek Hydrogeologic Regime \\ BWXT Y-12, L.L.C. \\ Chestnut Ridge Hydrogeologic Regime \\ calendar year \\ U.S. Department of Energy \\ Upper East Fork Poplar Creek Hydrogeologic Regime \\ U.S. Environmental Protection Agency \\ Groundwater Protection Program \\ Groundwater Monitoring Schedule \\ Monitoring and Remediation Optimization System \\ passive diffusion bag (sampler) \\ oxidation-reduction potential \\ volatile organic compounds \\ Y-12 National Security Complex
}




\subsection{INTRODUCTION}

This plan provides a description of the groundwater and surface water quality monitoring activities planned for calendar year (CY) 2008 at the U.S. Department of Energy (DOE) Y-12 National Security Complex (Y-12) that will be managed by the Y-12 Groundwater Protection Program (GWPP). Groundwater and surface water monitoring performed by the GWPP during CY 2008 will be in accordance with DOE Order 540.1 requirements and the following goals:

- to protect the worker, the public, and the environment;

- to maintain surveillance of existing and potential groundwater contamination sources;

- to provide for the early detection of groundwater contamination and determine the quality of groundwater and surface water where contaminants are most likely to migrate beyond the Oak Ridge Reservation property line;

- to identify and characterize long-term trends in groundwater quality at Y-12; and

- to provide data to support decisions concerning the management and protection of groundwater resources.

Groundwater and surface water monitoring during CY 2008 will be performed primarily in three hydrogeologic regimes at Y-12: the Bear Creek Hydrogeologic Regime (Bear Creek Regime), the Upper East Fork Poplar Creek Hydrogeologic Regime (East Fork Regime), and the Chestnut Ridge Hydrogeologic Regime (Chestnut Ridge Regime). The Bear Creek and East Fork regimes are located in Bear Creek Valley, and the Chestnut Ridge Regime is located south of Y-12 (Figure A.1). Additional surface water monitoring will be performed north of Pine Ridge, along the boundary of the Oak Ridge Reservation (Figure A.1).

Modifications to the CY 2008 monitoring program may be necessary during implementation. Changes in programmatic requirements may alter the analytes specified for selected monitoring wells or may add or remove wells from the planned monitoring network. All modifications to the monitoring program will be approved by the Y-12 GWPP manager and documented as addenda to this sampling and analysis plan.

The following sections of this report provide details regarding the CY 2008 groundwater and surface water monitoring activities. Section 2 describes the monitoring locations in each regime and the processes used to select the sampling locations. A description of the field measurements and laboratory analytes is provided in Section 3; sample collection methods and procedures are described in Section 4; and Section 5 lists the documents cited for more detailed operational and technical information.

The narrative sections of the report reference several appendices. Figures (maps and diagrams) and tables (excluding data summary tables presented in the narrative sections) are in Appendix A and Appendix B, respectively. Groundwater Monitoring Schedules (when issued) will be inserted in Appendix C, and addenda to this plan (if issued) will be inserted in Appendix D. Laboratory requirements (bottle lists, holding times, etc.) are provided in Appendix E. The updated sampling frequency for each monitoring well is in Appendix F, and an approved Waste Management Plan is provided in Appendix G. 


\subsection{MONITORING LOCATIONS}

The monitoring locations to be sampled by the Y-12 GWPP during CY 2008 (Table B.1) were selected based on results of a comprehensive assessment of the Y-12 GWPP using the Monitoring and Remediation Optimization System (MAROS) software (BWXT Y-12, L.L.C. [BWXT] 2005). The MAROS assessment provided recommendations (e.g., sampling frequency) for the monitoring locations with sufficient analytical data obtained between CY 1996 and CY 2004. The monitoring wells selected for sample collection in CY 2008 include semiannual, annual, and biennial sampling frequencies (Appendix F). The sampling frequency for each location reflects subsequent evaluation of each MAROS recommendation including monitoring results obtained after CY 2004, as described in the Y-12 GWPP Monitoring Optimization Plan (BWXT 2006a). Additionally, the sampling frequency has been modified for 38 of the CY 2008 sampling wells, including a reduced frequency for 18 wells and an increased frequency for 20 wells (Appendix F). Reducing the sampling frequency for these wells is based on recommendations in the CY 2006 Groundwater Monitoring Report (BWXT 2007a). The increased sampling frequency is for 14 monitoring wells that were selected for review from the "to be determined" category in the Monitoring Optimization Plan and six wells that need results for at least four groundwater samples by CY 2010 to be included in a subsequent MAROS assessment.

The Y-12 GWPP monitoring network for CY 2008 includes 138 monitoring locations (Table B.1): 50 located in the Bear Creek Regime (Figure A.2), 13 located in the Chestnut Ridge Regime (Figure A.3), 71 located in the East Fork Regime (Figure A.4), and three located north of Pine Ridge (Figure A.5). Groundwater samples will be collected from a total of 124 monitoring wells, including 45 wells in the Bear Creek Regime (Figure A.2), eight wells in the Chestnut Ridge Regime, and 71 wells in the East Fork Regime (Figure A.4). Well GW-722, located in the East Fork Regime, contains a Westbay ${ }^{\mathrm{TM}}$ multiport sampling system and is scheduled for sample collection from five ports at different depths in the well (Figure A.6). Samples of groundwater discharging from four natural springs will be collected during CY 2008, including two springs (SS-4 and SS-5) in the Bear Creek Regime (Figure A.2) and two springs (SCR2.1SP and SCR2.2SP) in the Chestnut Ridge Regime (Figure A.3).

Surface water samples will be collected from a total of nine sampling locations during CY 2008, including two locations in the Bear Creek Regime, three locations in the Chestnut Ridge Regime, and four locations north of Pine Ridge. In the Bear Creek Regime, samples will be collected from Bear Creek at one sampling station located from about 4.5 kilometers upstream of the confluence of Bear Creek and East Fork Poplar Creek (BCK-04.55) and from one sampling station along a northern tributary (NT-01) to Bear Creek (Figure A.2). The tributaries located in the Chestnut Ridge Regime have been numbered from west to east (SCR1 through SCR5) and surface water samples will be collected from three of the tributaries at stations (SCR1.5SW, SCR3.5SW, and S17 [located in SCR5]) located along the north side of Bethel Valley Road (Figure A.3). The surface water sampling locations north of Pine Ridge include two tributaries (NPR12.0SW and NPR23.0SW) near the Scarboro Community and one location (GHK2.51ESW) near Country Club Estates (Figure A.5). 


\subsection{FIELD MEASUREMENTS AND ANALYTICAL PARAMETERS}

Before collecting samples at each monitoring location, field personnel will record (on Field Data Sheets) the following field measurements (Table B.2):

- depth to the static water level in monitoring wells;

- $\mathrm{pH}$;

- water temperature;

- conductivity;

- dissolved oxygen; and

- oxidation-reduction potential (REDOX)

Field measurement of dissolved oxygen and REDOX will not be obtained for sampling ports of monitoring wells equipped with a Westbay ${ }^{\mathrm{TM}}$ multiport sampling system. Instead of measuring the depth to the static water level in each Westbay ${ }^{\mathrm{TM}}$ sampling zone, the potentiometric head (in $\mathrm{ft}$ ) will be calculated from subsurface pressure measurements obtained.

For this Sampling and Analysis Plan, specific analytes are grouped by analytical method or by type (e.g., trace metals) and referenced as parameter groups (Table B.1 and Table B.2). In addition to field measurements, all groundwater and surface water samples will be analyzed for the following suite of parameters (identified as the Standard Administrative Parameter Group):

- miscellaneous laboratory analytes (turbidity, total suspended solids and total dissolved solids);

- major anions;

- trace metals (includes major cations);

- a comprehensive suite of volatile organic compounds (VOCs); and

- gross alpha and gross beta activity.

In addition to the analytes included in the Standard Administrative Parameter Group, samples from selected locations will be analyzed for specific radionuclides and/or methanol. The radionuclide analyses will supplement gross alpha and/or gross beta activity results, especially in cases where the gross activity reporting limits are elevated from interferences caused by a high dissolved solid content of the groundwater sample (see Appendix E). 


\subsection{SAMPLE PLANNING, COLLECTION, AND HANDLING}

Previous Y-12 GWPP Sampling and Analysis Plans assembled the monitoring locations into sample groups (e.g., BC-1) for sample collection, sample tracking, and data management purposes. However, using dedicated sampling equipment and varying sampling frequencies over time have made the sample groups unnecessary. The monitoring locations will continue to be grouped by hydrogeologic regime to provide manageable geographic areas for planning purposes, and the specific CY quarter for sample collection is provided for each monitoring location (Table B.1).

A Groundwater Monitoring Schedule (GWMS) will be prepared by GWPP personnel for each quarterly sampling event of CY 2008. Each GWMS (four per year) will be issued before sample collection begins, will specify the sequence for collecting samples from the monitoring locations scheduled for the quarter, and will include information necessary for field personnel to collect the required samples (e.g., containment requirements and previous pumping rates used to sample each well). The GWMS is considered to be an integral part of this document; when issued, the GWMS for each CY 2008 quarterly sampling event will be inserted in Appendix C.

Unfiltered samples will be collected semiannually (46 samples) or annually (114 samples, including 20 biennial samples) from the monitoring locations during CY 2008. As summarized below, the number of samples to be collected during each CY quarter will range from 33 to 45, for an annual total of 160 samples.

\begin{tabular}{|c|c|c|c|c|}
\hline \multirow{2}{*}{$\begin{array}{c}\text { HYDROGEOLOGIC } \\
\text { REGIME/AREA }\end{array}$} & \multicolumn{4}{|c|}{ NuMBER OF SAMPLES PER QUARTER OF CY 2008 } \\
\cline { 2 - 5 } & $\mathbf{1 s t}$ & 2nd & 3rd & 4th \\
\hline Bear Creek Regime & 37 & 0 & 28 & 0 \\
Chestnut Ridge Regime & 0 & 6 & 0 & 8 \\
East Fork Regime & 1 & 39 & 16 & 22 \\
North of Pine Ridge & 0 & 0 & 0 & 3 \\
\hline TOTAL: & $\mathbf{3 8}$ & $\mathbf{4 5}$ & $\mathbf{4 4}$ & $\mathbf{3 3}$ \\
\hline
\end{tabular}

Personnel from the Sampling Services Section of the Y-12 Environment Compliance Department will be responsible for collection, transportation, and chain-of-custody control of all groundwater and surface water samples. Based on the analytical parameters for the CY 2008 monitoring locations (Table B.1 and Table B.2), personnel with the Y-12 Analytical Chemistry Organization (ACO) will prepare bottle lists that specify the sample container type, size, preservative, and the laboratory test identification needed for each sampling location (see Appendix E). Additionally, ACO personnel will generate a weekly tracking report to record the sample collection date and time for each monitoring location, and the date that analyses are scheduled for completion or if analysis is completed. Sample collection will be performed in accordance with the most recent version of operating procedures for obtaining groundwater samples (BWXT 2002a, BWXT 2004, BWXT 2006b, and BWXT 2007b) and surface water samples (BWXT 2002b). All field and laboratory activities will be performed in accordance with applicable requirements of the Y-12 Integrated Safety Management System and task-specific job hazard analyses.

Groundwater samples will be collected using the low-flow minimal drawdown method (low-flow method) during CY 2008 from all monitoring wells, except for one well (GW-722) equipped with a Westbay ${ }^{\mathrm{TM}}$ multiport sampling system. For the low-flow method, a bladder pump is permanently installed in each well that is scheduled for sample collection. If well construction prevents permanent installation (e.g., flushmounted wells), then the pump and tubing will be installed at least 24 hours before sample collection and will 
be removed when sampling is completed. In accordance with the groundwater sampling procedure for the low-flow method (BWXT 2007b), groundwater is purged, and subsequently sampled, from the well at a flow rate ( $<300$ milliliters per minute) which ensures minimal drawdown of the static water level, therefore isolating the stagnant water column above the intake of the pump. Groundwater samples are collected from a well after the water level is in steady-state drawdown $(<0.1 \mathrm{ft}$ over a 15 -minute interval) and field parameters ( $\mathrm{pH}$, conductivity, water temperature, REDOX, and dissolved oxygen) have stabilized (minimal variation over four consecutive readings).

Groundwater sampling and pressure profiling using a Westbay ${ }^{\mathrm{TM}}$ multiport sampling system at well GW-722 in the East Fork Regime will be performed in accordance with the operating procedures (BWXT 2002a and BWXT 2006b). The groundwater samples from each sampling port (Figure A.6) will be collected in 250-milliliter nonvented stainless steel Westbay ${ }^{\mathrm{TM}}$ sample collection bottles filled at the designated depth in the well. Once filled, the bottles will be raised to the surface and the groundwater will be transferred to laboratory sample containers. The sample collection bottles will be lowered, filled, and retrieved as many times as needed to completely fill the laboratory sample bottles. Groundwater in the first sample collection bottles retrieved from each sampling port will be used as a "formation rinse" to obtain field measurements and to condition the sample collection bottle for each zone.

In conjunction with the low-flow sampling method, passive diffusion bag (PDB) samplers also will be used to evaluate VOC concentrations in groundwater at two wells (GW-322 and GW-612) located at the Chestnut Ridge Security Pits (Table B.1). A PDB is polyethylene bag (semipermeable membrane) that is filled with deionized water, lowered to the monitored interval of the well, and remains in the well for at least two weeks to allow VOC concentrations in the bag to reach equilibrium (passive diffusion) with the surrounding groundwater. After retrieval, sample bottles for VOC analyses will be filled with water from the PDB. Analytical results for the PDB samplers (and collection costs) will be compared to the low-flow sampling results to determine if using PDBs is acceptable in areas where VOCs are the only contaminant of concern.

In addition to the groundwater and surface water samples, field blanks and equipment rinsate samples will be collected at the frequencies and analyzed for the parameter groups specified on Table B.1. Field blank samples will be collected for at least $1 \%$ of the samples. Therefore, two field blank samples will be collected during CY 2008: in the East Fork Regime during the second quarter and in the Chestnut Ridge Regime during the fourth quarter. An equipment rinsate sample will be collected from Westbay well GW-722 (Table B.1) immediately after field-cleaning the sampling equipment used to collect samples from the last sampling port (GW-722-17).

Trip blank samples and field duplicate samples will be prepared and handled in accordance with the Field Quality Control Samples operating procedure (BWXT 2007c) and will be analyzed using applicable procedures. Trip blank samples will be prepared for each cooler used to transport samples for volatile organic analyses. Because duplicate samples will be collected from at least $10 \%$ of the sampling locations, a total of 16 field duplicate samples will be collected during CY 2008: six in the Bear Creek Regime, two in the Chestnut Ridge Regime, and eight in the East Fork Regime (Table B.1).

All groundwater and surface water samples will be relinquished under chain-of-custody control to the appropriate Y-12 ACO laboratory that will perform the analyses. The Y-12 ACO laboratories will perform each analyses within established holding times and deliver results in hard copy and electronic format within established turnaround times (see Appendix E). 


\subsection{REFERENCES}

American Public Health Association. 1992. Standard Methods for Examination of Water and Wastewater, $18^{\text {th }}$ Edition.

BWXT Y-12, L.L.C. 2002a. Pressure Profiling of Wells Equipped with Westbay ${ }^{\mathrm{TM}}$ Monitoring System Instrumentation. BWXT Y-12, L.L.C. Management Requirement prepared by the Environment, Safety, and Health Organization (Y50-71-019, Rev.1).

BWXT Y-12, L.L.C. 2002b. Liquid Grab Sampling. BWXT Y-12, L.L.C. Management Requirement prepared by the Environment, Safety, and Health Organization (Y50-71-005, Rev. 1.1).

BWXT Y-12, L.L.C. 2004. Measurement of Static Water Level Elevation. BWXT Y-12, L.L.C. Management Requirement prepared by the Environment, Safety, and Health Organization (Y50-71-015, Rev 1.0).

BWXT Y-12, L.L.C. 2005. Assessment of the Groundwater Protection Program, Y-12 National Security Complex, Oak Ridge, Tennessee. Prepared by Groundwater Services, Inc. (Y/TS-1984).

BWXT Y-12, L.L.C. 2006a. Y-12 Groundwater Protection Program Monitoring Optimization Plan for Groundwater Monitoring Wells at the U.S. Department of Energy Y-12 National Security Complex, Oak Ridge, Tennessee. Prepared by Elvado Environmental LLC (Y/TS-2031).

BWXT Y-12, L.L.C. 2006b. Groundwater Sampling of Westbay ${ }^{\mathrm{TM}}$ Monitoring System Instrumented Wells. BWXT Y-12, L.L.C. Management Requirement prepared by the Environment, Safety, and Health Organization (Y50-71-018, Rev.2).

BWXT Y-12, L.L.C. 2007a. Calendar Year 2006 Groundwater Monitoring Report, U.S. Department of Energy Y-12 National Security Complex, Oak Ridge, Tennessee. Prepared by Elvado Environmental LLC (Y/SUB/07-054638/1).

BWXT Y-12, L.L.C. 2007b. Groundwater Sampling. BWXT Y-12, L.L.C. Management Requirement prepared by the Environment, Safety, and Health Organization (Y50-71-016, Rev 2.0).

BWXT Y-12, L.L.C. 2007c. Field Quality Control Samples. BWXT Y-12, L.L.C. Management Requirement prepared by the Environment, Safety, and Health Organization (Y71-66-EC-003, Rev 2.0).

U.S. Environmental Protection Agency. 1983. Methods for Chemical Analysis of Water and Wastes.

U.S. Environmental Protection Agency. 1996. Test Methods for Evaluating Solid Waste Physical/Chemical Methods. 
APPENDIX A

\section{FIGURES}




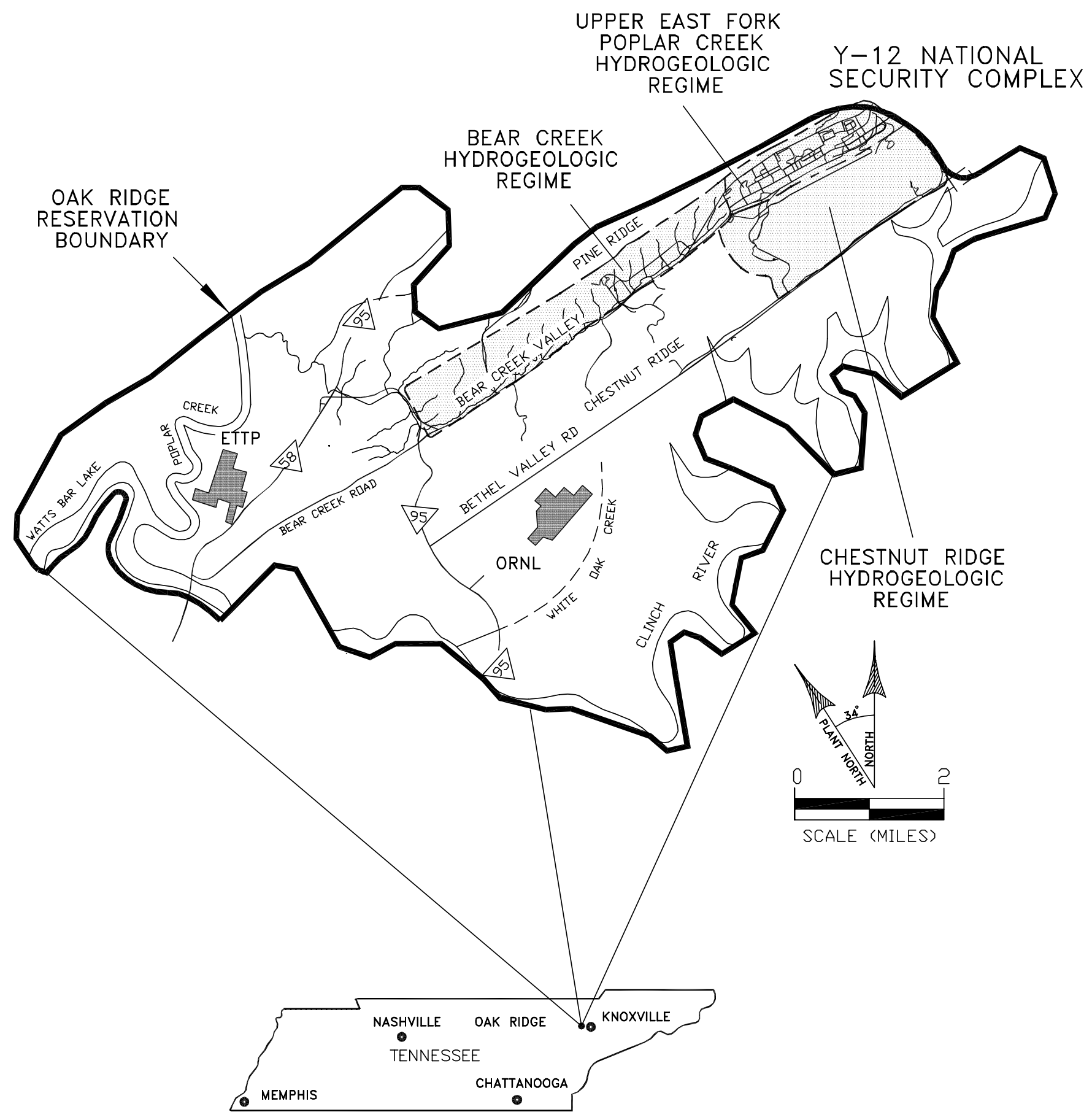

Fig. A.1. Hydrogeologic regimes at the Y-12 National Security Complex. 


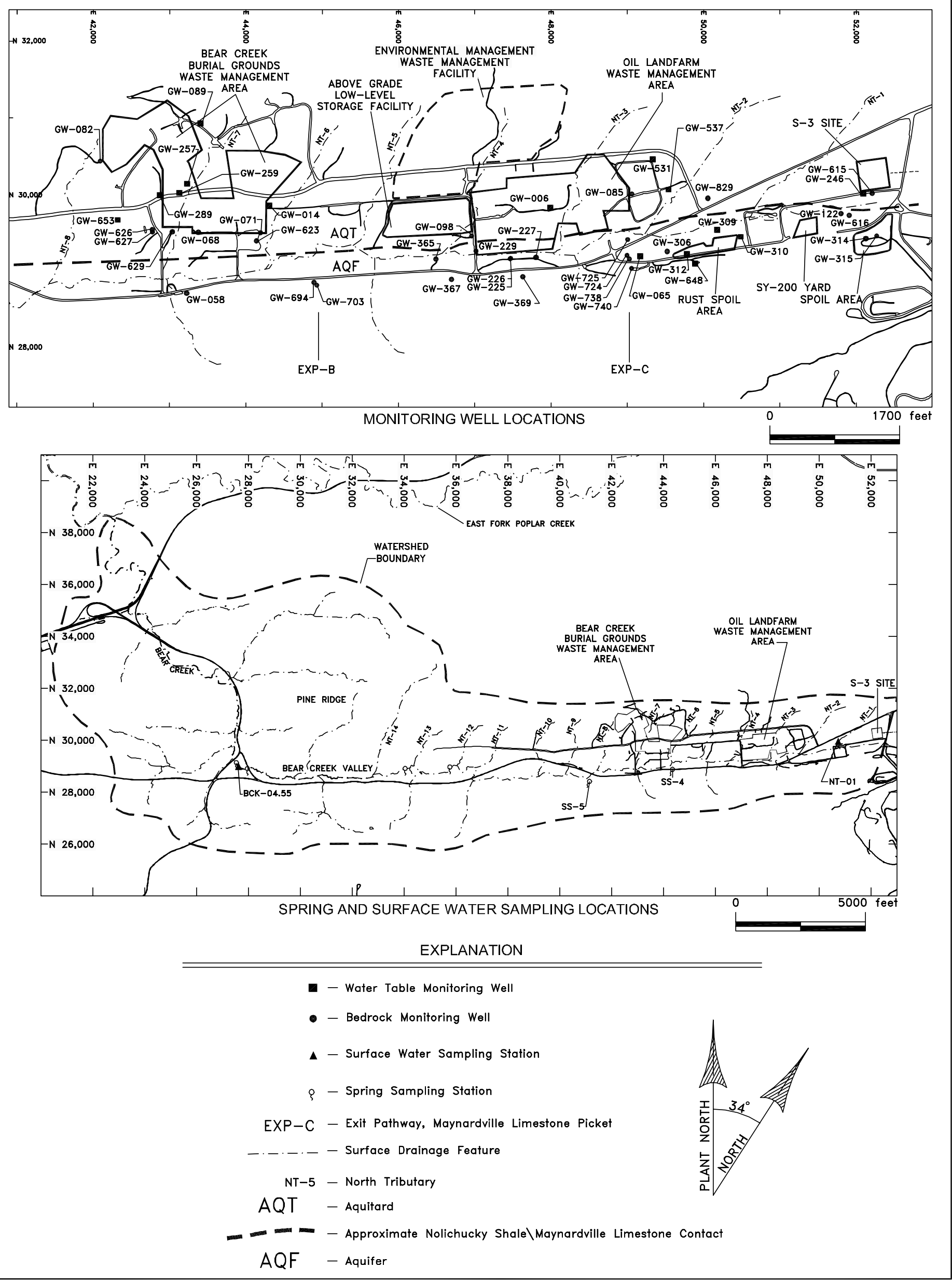




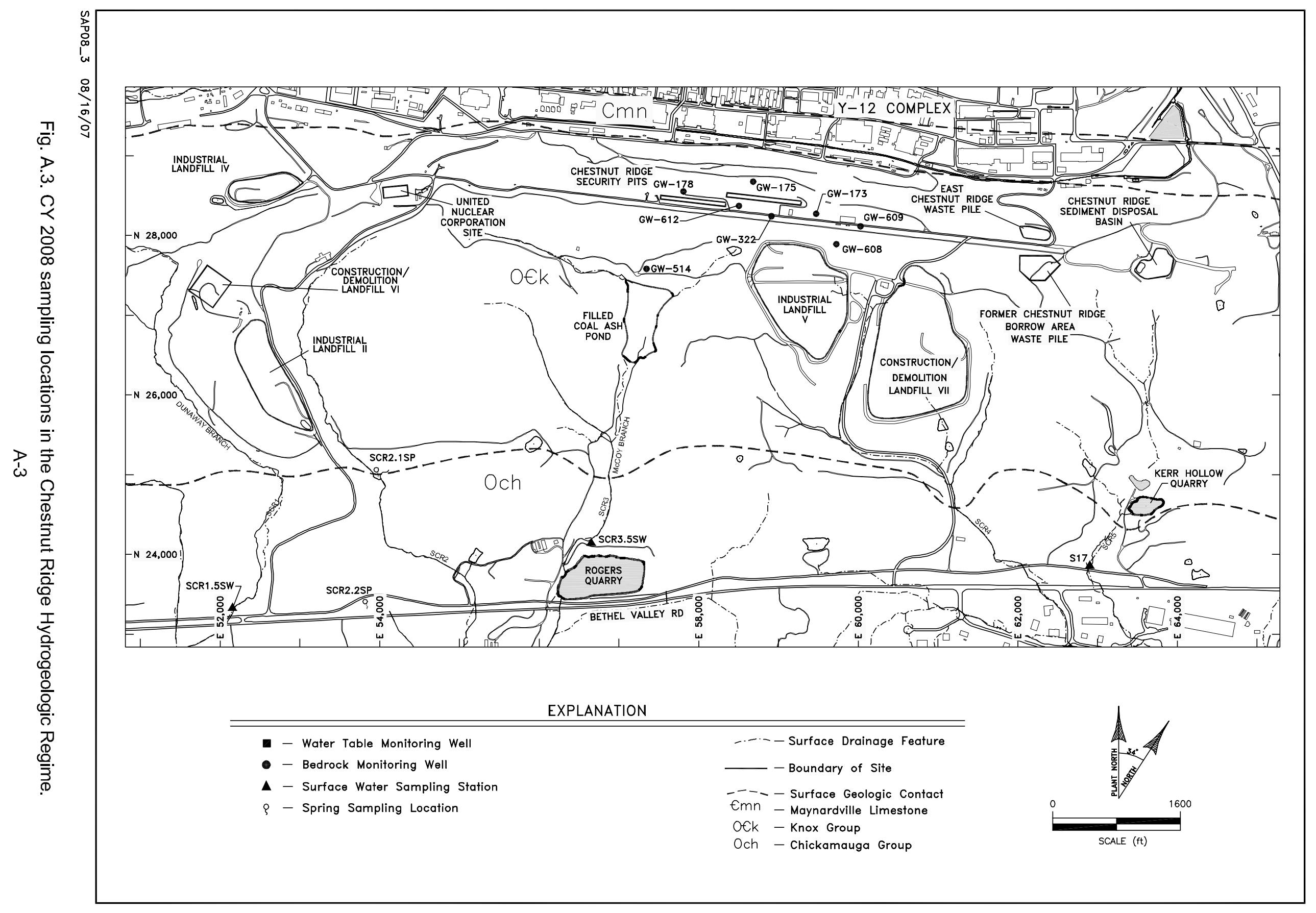




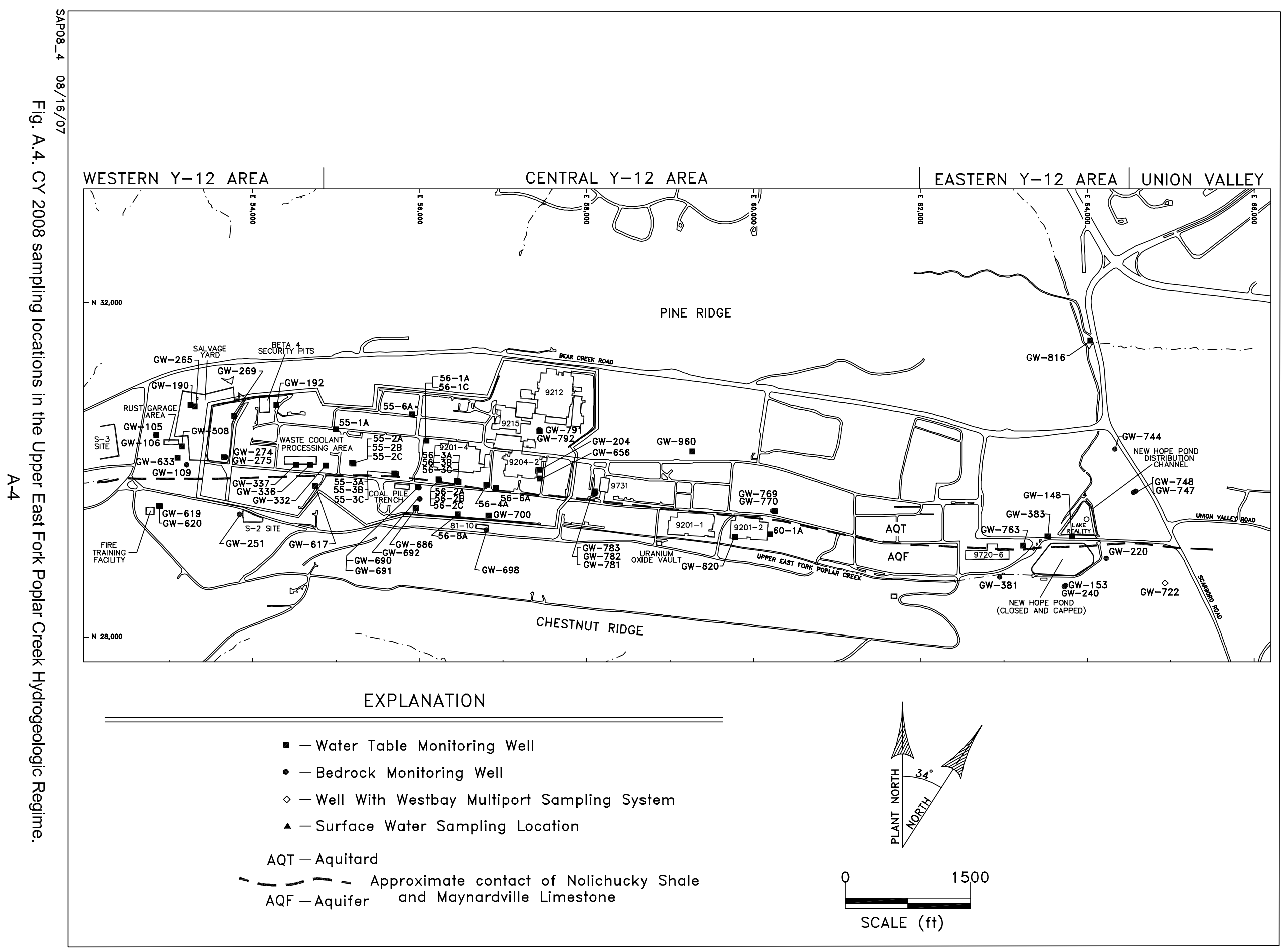




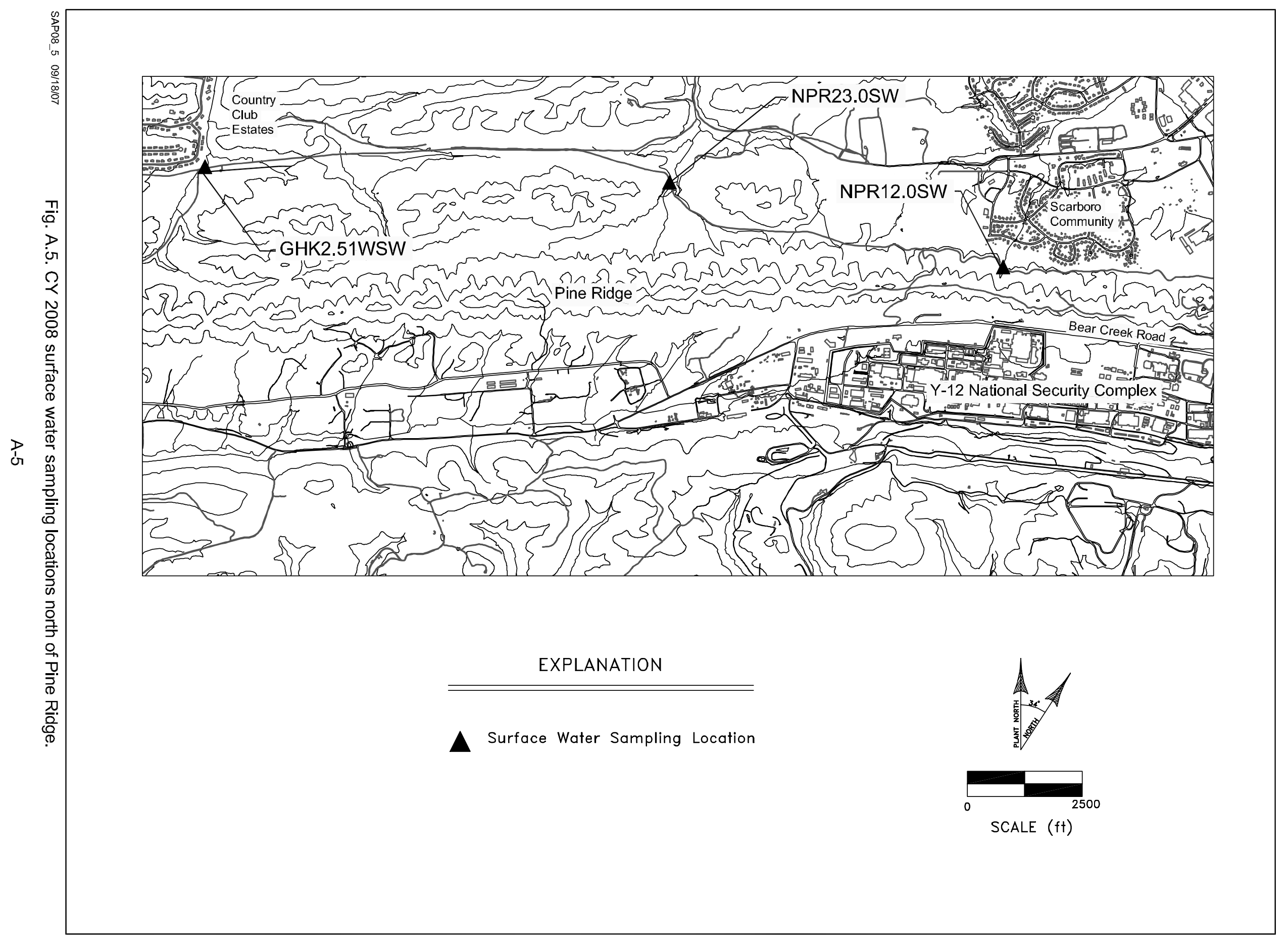




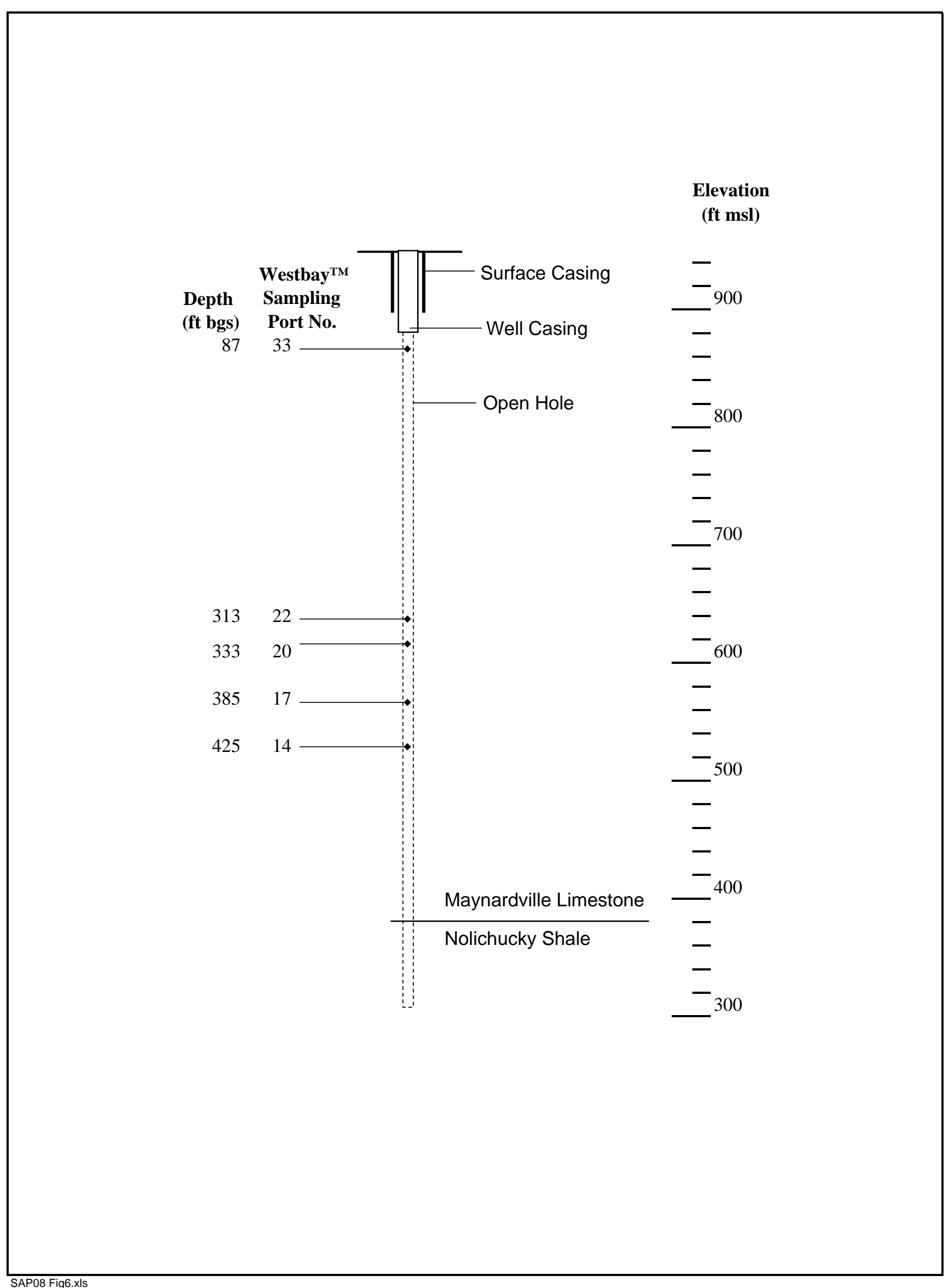

Fig. A.6. Westbay ${ }^{\mathrm{TM}}$ monitoring system sampling port depths in well GW-722. 
APPENDIX B

TABLES 
Table B.1 Sampling locations, frequency, and analytical parameters for groundwater and surface water monitoring during CY 2008

\begin{tabular}{|c|c|c|c|c|c|c|c|c|}
\hline \multirow{2}{*}{$\begin{array}{c}\text { Sampling } \\
\text { Point }^{1}\end{array}$} & \multirow{2}{*}{ Location $^{2}$} & \multirow{2}{*}{$\begin{array}{c}\text { Hydrostrat. } \\
\text { Unit }^{3} \\
\end{array}$} & \multirow{2}{*}{$\begin{array}{c}\text { Tag } \\
\text { Depth }^{4}\end{array}$} & \multicolumn{4}{|c|}{ Samples Collected in CY $2008^{5}$} & \multirow{2}{*}{ Parameters $^{6}$} \\
\hline & & & & Q1 & Q2 & Q3 & Q4 & \\
\hline \multicolumn{9}{|c|}{ Bear Creek Hydrogeologic Regime } \\
\hline $\mathrm{GW}-006$ & OLF & Aquitard & 51.08 & Y & & Y & & STD \\
\hline GW-014 & BG & Aquitard & 14.50 & & & Y & & STD \\
\hline GW-058 & BG & Aquifer & 48.90 & Y & & & & STD \\
\hline GW-065 & OLF & Aquifer & 36.89 & Y & & Y & & STD \\
\hline GW-068 & $\mathrm{BG}$ & Aquitard & 86.10 & & & Y & & STD \\
\hline $\mathrm{GW}-071$ & $\mathrm{BG}$ & Aquitard & 218.40 & $\mathrm{Y}$ & & $\mathrm{Y}$ & & STD \\
\hline GW-082 & $\mathrm{BG}$ & Aquitard & 38.45 & & & Y & & STD \\
\hline GW-085 & OLF & Aquitard & 62.34 & Y & & Y & & STD \\
\hline GW-089 & $\mathrm{BG}$ & Aquitard & 27.97 & & & $Y$ & & STD \\
\hline GW-098 & OLF & Aquitard & 105.65 & Y & & & & STD \\
\hline $\mathrm{GW}-122$ & S3 & Aquifer & 145.28 & Y & & & & STD \\
\hline GW-225 & $\mathrm{OLF}$ & Aquifer & 203.30 & $\mathrm{Y}$ & & $\mathrm{Y}$ & & STD \\
\hline GW-226 & OLF & Aquifer & 58.47 & Y & & Y & & STD \\
\hline GW-227 & OLF & Aquifer & 42.64 & Y & & & & STD \\
\hline GW-229 & $\mathrm{OLF}$ & Aquifer & 51.45 & $\mathrm{D}$ & & & & STD \\
\hline GW-246 & S3 & Aquitard & 76.50 & Y & & Y & & STD, RAD $(3,12)$ \\
\hline GW-257 & BG & Aquitard & 36.63 & & & Y & & STD \\
\hline GW-259 & $\mathrm{BG}$ & Aquitard & 35.74 & Y & & Y & & STD \\
\hline GW-289 & $\mathrm{BG}$ & Aquitard & 43.14 & & & Y & & STD \\
\hline GW-306 & RS & Aquifer & 60.66 & Y & & Y & & STD, RAD(12) \\
\hline GW-309 & RS & Aquifer & 40.06 & Y & & $\mathrm{D}$ & & STD, RAD(12) \\
\hline GW-310 & $\mathrm{RS}$ & Aquifer & 30.47 & Y & & & & STD, RAD(12) \\
\hline GW-312 & RS & Aquifer & 42.10 & Y & & & & STD \\
\hline $\mathrm{GW}-314$ & SPI & Aquifer & 118.15 & Y & & $\mathrm{Y}$ & & STD \\
\hline GW-315 & SPI & Aquifer & 105.98 & $Y$ & & & & STD \\
\hline GW-365 & OLF & Aquifer & 152.49 & Y & & & & STD \\
\hline GW-367 & OLF & Aquifer & 153.48 & Y & & Y & & STD \\
\hline GW-369 & OLF & Aquifer & 150.30 & $\mathrm{D}$ & & & & STD \\
\hline $\mathrm{GW}-531$ & LD & Aquitard & 41.28 & & & $\mathrm{Y}$ & & STD \\
\hline GW-537 & OLF & Aquitard & 27.35 & & & Y & & STD, RAD(12) \\
\hline GW-615 & S3 & Aquitard & 246.84 & & & Y & & STD, RAD $(3,12)$ \\
\hline GW-616 & S3 & Aquitard & 270.59 & & & $\mathrm{Y}$ & & STD \\
\hline GW-623 & BG & Aquitard & 277.93 & $\mathrm{Y}$ & & Y & & STD \\
\hline GW-626 & $\mathrm{BG}$ & Aquitard & 80.92 & Y & & Y & & STD \\
\hline GW-627 & $\mathrm{BG}$ & Aquitard & 270.96 & Y & & Y & & STD \\
\hline GW-629 & $\mathrm{BG}$ & Aquitard & 314.59 & Y & & Y & & STD \\
\hline GW-648 & $\mathrm{RS}$ & Aquifer & 82.47 & Y & & & & STD \\
\hline GW-653 & BG & Aquitard & 41.53 & & & $\mathrm{D}$ & & STD \\
\hline GW-694 & EXP-B & Aquifer & 207.27 & Y & & & & STD \\
\hline GW-703 & EXP-B & Aquifer & 185.29 & Y & & & & STD \\
\hline GW-724 & EXP-C & Aquifer & 293.60 & Y & & & & STD \\
\hline GW-725 & EXP-C & Aquifer & 145.42 & $\mathrm{D}$ & & & & STD \\
\hline GW-738 & EXP-C & Aquifer & 91.78 & Y & & & & STD \\
\hline GW-740 & EXP-C & Aquifer & 192.67 & Y & & & & STD \\
\hline GW-829 & OLF & Aquitard & 118.68 & & & $\mathrm{Y}$ & & $\operatorname{STD}, \operatorname{RAD}(3,12)$ \\
\hline
\end{tabular}


Table B.1 (continued)

\begin{tabular}{|c|c|c|c|c|c|c|c|c|}
\hline \multirow{2}{*}{$\begin{array}{c}\text { Sampling } \\
\text { Point }^{1} \\
\end{array}$} & \multirow{2}{*}{ Location $^{2}$} & \multirow{2}{*}{$\begin{array}{c}\text { Hydrostrat. } \\
\text { Unit }^{3}\end{array}$} & \multirow{2}{*}{$\begin{array}{c}\text { Tag } \\
\text { Depth }^{4}\end{array}$} & \multicolumn{4}{|c|}{ Samples Collected in CY $2008{ }^{5}$} & \multirow{2}{*}{ Parameters $^{6}$} \\
\hline & & & & Q1 & Q2 & Q3 & Q4 & \\
\hline \multicolumn{9}{|c|}{ Bear Creek Hydrogeologic Regime (continued) } \\
\hline BCK-04.55 & EXP-SW & & & Y & & & & STD \\
\hline NT-01 & EXP-SW & & & Y & & & & STD \\
\hline SS-4 & EXP-SW & & & 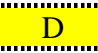 & & & & STD \\
\hline SS-5 & EXP-SW & .". & $\cdot$ & Y & & & & STD \\
\hline \multicolumn{9}{|c|}{ Chestnut Ridge Hydrogeologic Regime } \\
\hline GW-173 & CRSP & Aquifer & 167.34 & & Y & & $\mathrm{D}$ & STD \\
\hline $\mathrm{GW}-175$ & CRSP & Aquifer & 169.49 & & & & Y... & STD \\
\hline GW-178 & CRSP & Aquifer. & 134.68 & & & & Y... & STD \\
\hline FB-GW-322 & CRSP & & & & & & Y & VOC(1) \\
\hline GW-322 & CRSP & Aquifer & 191.99 & & & & $\mathrm{Y}^{*}$ & STD \\
\hline GW-514 & FCAP & Aquifer & 197.13 & & & & Y & STD \\
\hline GW-608 & CRSP & Aquifer & 219.80 & & & & Y & STD \\
\hline GW-609 & CRSP & Aquifer & 268.80 & & & & Y & STD \\
\hline GW-612 & CRSP & Aquifer & 256.28 & & & & $\mathrm{Y}^{*}$ & STD \\
\hline S17 & EXP-SW & & & & Y & & & STD \\
\hline SCR1.5SW & EXP-SW & & & & Y & & & STD" \\
\hline SCR2.1SP & EXP-SW & & & & Y & & & STD \\
\hline SCR2.2SP & EXP-SW & & & & $\mathrm{D}$ & & & STD \\
\hline SCR3.5SW & EXP-SW & . &. & & $\mathrm{Y}$ & & & STD \\
\hline \multicolumn{9}{|c|}{ Upper East Fork Poplar Creek Hydrogeologic Regime } \\
\hline $55-1 \mathrm{~A}$ & GRIDB2 & Aquitard & 19.22 & & Y & & & STD \\
\hline $55-2 \mathrm{~A}$ & GRIDB3 & Aquitard & 13.98 & & Y.... & & & STD, VOC(3) \\
\hline $55-2 \mathrm{~B}$ & GRIDB3 & Aquitard & 27.69 & & $\mathrm{D}$ & & & STD, VOC(3) \\
\hline $55-2 \mathrm{C}$ & GRIDB3 & Aquitard & 76.00 & & Y & & & STD, VOC(3) \\
\hline FB-55-3A & B9201-5 & & & & 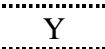 & & & VOC (1) \\
\hline $55-3 \mathrm{~A}$ & B9201-5 & Aquitard & 14.25 & & $\mathrm{Y}$ & & & STD, VOC(3) \\
\hline 55-3B & B9201-5 & Aquitard & 37.98 & & Y'."' & & & STD, VOC(3) \\
\hline $55-3 \mathrm{C}$ & B9201-5 & 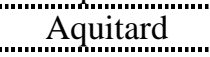 & 77.43 & & 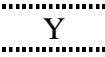 & & & STD, VOC(3) \\
\hline $55-6 A$ & B9103 & Aquitard" & 12.77 & & $\mathrm{Y}$ & & & STD \\
\hline $56-1 \mathrm{~A}$ & Y12 & Aquitard & 18.95 & & Y & & & STD \\
\hline $56-1 C$ & Y12 & Aquitard & 73.45 & & $\mathrm{Y}$ & & & STD \\
\hline $56-2 A$ & GRIDC3 & Aquitard & 15.03 & & $\mathrm{Y}$ & & & STD, VOC(3) \\
\hline $56-2 B$ & GRIDC3 & Aquitard & 38.63 & & Y & & & STD, VOC(3) \\
\hline $56-2 \mathrm{C}$ & GRIDC3 & Aquitard & 77.03 & & $Y$ & & & STD, VOC(3) \\
\hline $56-3 \mathrm{~A}$ & Y12 & Aquitard & 17.92 & & Y & & & STD, VOC(3) \\
\hline $56-3 B$ & Y12 & Aquitard & 30.85 & & $\mathrm{D}$ & & & STD, VOC(3) \\
\hline $56-3 \mathrm{C}$ & $\mathrm{Y} 12$ & Aquitard & 55.35 & & $\mathrm{Y}$ & & & STD, VOC(3) \\
\hline $56-4 A$ & Y12 & Aquitard & 12.60 & & Y'.' & & & STD, VOC(3) \\
\hline $56-6 A$ & Y12 & Aquitard & 20.97 & & Y'" & & & STD, VOC(3) \\
\hline $56-8 \mathrm{~A}$ & Y12 & Aquifer & 25.44 & & Y & & & STD \\
\hline $60-1 A$ & Y12" & Aquifer & 23.10 & & & & $\mathrm{Y}$ & STD" \\
\hline GW-105 & S3 & Aquitard & 19.40 & & $\mathrm{Y}$ & & & STD \\
\hline GW-106 & S3 & Aquitard & 74.10 & & $\mathrm{D}$ & & & STD \\
\hline GW-109 & S3 & Aquitard & 125.45 & & $\mathrm{Y}$ & & & STD, RAD $(3,12)$ \\
\hline GW-148 & NHP & Aquifer & 13.93 & & & $\mathrm{Y}$ & & STD \\
\hline GW-153 & NHP & Aquifer & 60.84 & & & Y & & STD \\
\hline GW-190 & B4 & Aquitard & 29.84 & & $\mathrm{Y}$ & & & STD \\
\hline $\mathrm{GW}-192$ & B4 & Aquitard & 21.58 & & $\mathrm{Y}$ & & & STD \\
\hline
\end{tabular}


Table B.1 (continued)

\begin{tabular}{|c|c|c|c|c|c|c|c|c|}
\hline \multirow{2}{*}{\begin{tabular}{|c|} 
Sampling \\
Point $^{1}$ \\
\end{tabular}} & \multirow{2}{*}{ Location $^{2}$} & \multirow{2}{*}{$\begin{array}{c}\text { Hydrostrat. } \\
\text { Unit }^{3} \\
\end{array}$} & \multirow{2}{*}{$\begin{array}{c}\text { Tag } \\
\text { Depth }^{4}\end{array}$} & \multicolumn{4}{|c|}{ Samples Collected in CY $2008{ }^{5}$} & \multirow{2}{*}{ Parameters ${ }^{6}$} \\
\hline & & & & Q1 & Q2 & Q3 & Q4 & \\
\hline \multicolumn{9}{|c|}{ Upper East Fork Poplar Creek Hydrogeologic Regime (continued) } \\
\hline GW-204 & T0134 & Aquitard & 20.23 & & & & $\mathrm{Y}$ & STD \\
\hline GW-220 & NHP & Aquifer & 49.00 & Y & & $\mathrm{D}$ & & STD \\
\hline GW-240" & NHP & Aquifer & 32.55 & & & 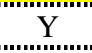 & & STD \\
\hline $\mathrm{GW}-251$ & S2 & Aquifer & 50.04 & & 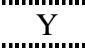 & & & STD \\
\hline $\mathrm{GW}-265$ & SY & Aquitard & 25.68 & & Y.." & & & STD \\
\hline GW-269 & SY & Aquitard & 33.50 & & Y... & & & STD \\
\hline GW-274 & SY & Aquitard & 36.12 & & $\mathrm{Y}$ & & & STD, RAD $(3,12)$ \\
\hline GW-275 & SY & Aquitard & 68.47 & & $\mathrm{Y}$ & & & $\mathrm{STD}, \mathrm{RAD}(3,12)$ \\
\hline GW-332 & WC & Aquitard & 27.07 & & Y.e & & & STD \\
\hline GW-336 & WC & Aquitard & 23.93 & & Y & & & STD \\
\hline GW-337" & WC & Aquitard & 25.33 & & Y.'." & & & STD \\
\hline GW-381 & NHP & Aquifer & 61.01 & & & Y... & & STD \\
\hline GW-383 & NHP & Aquitard & 26.54 & & & Y & & STD \\
\hline GW-508 & $\mathrm{RG}$ & Aquitard & 15.11 & & $\mathrm{Y}$ & & $\mathrm{Y}$ & STD \\
\hline GW-617" & EXP-E & Aquifer & 20.69 & & & & 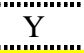 & STD \\
\hline GW-619 & FTF & Aquifer & 43.63 & & & & D & STD, RAD(12) \\
\hline GW-620 & FTF & Aquifer & 77.91 & & & & $\mathrm{Y}$ & STD \\
\hline GW-633 & $\mathrm{RG}$ & Aquitard & 15.15 & & Y & & & STD, RAD(12) \\
\hline GW-656 & T0134 & Aquitard & 20.60 & & & & Y & STD \\
\hline GW-686 & СРT & Aquifer & 16.23 & & & & Y & STD \\
\hline GW-690 & СPT & Aquifer & 53.25 & & & & Y & STD \\
\hline GW-691 & CPT & Aquifer & 20.39 & & & & Y & STD \\
\hline GW-692 & $\mathrm{CPT}$ & Aquifer & 53.05 & & & & Y & STD \\
\hline GW-698 & B8110 & Aquifer & 74.88 & & $\mathrm{D}$ & & Y & STD \\
\hline GW-700 & B8110 & Aquifer & 33.19 & & & & Y & STD \\
\hline GW-722-14 & EXP-J & Aquifer & 425.00 & & & $\mathrm{Y}$ & & STD \\
\hline GW-722-17 & EXP-J & Aquifer & 385.00 & & & 紧 & & STD \\
\hline ER-GW-722-17 & EXP-J & & & & & Y & & VOC(1) \\
\hline GW-722-20 & EXP-J & Aquifer & 333.00 & & & $\mathrm{Y}$ & & STD \\
\hline GW-722-22 & EXP-J & Aquifer & 313.00 & & & Y & & STD \\
\hline GW-722-33 & EXP-J & Aquifer & 87.00 & & & D & & STD \\
\hline GW-744 & GRIDK1 & Aquitard & 69.28 & & & Y & & STD \\
\hline GW-747 & GRIDK2 & Aquitard & 82.33 & & & Y & & STD \\
\hline GW-748 & GRIDK2 & Aquitard & 29.80 & & & Y & & STD \\
\hline GW-763 & GRIDJ3 & Aquitard & 20.41 & & & Y & & STD \\
\hline GW-769 & GRIDG3 & Aquitard & 62.73 & & Y & & Y & STD \\
\hline GW-770 & GRIDG3 & Aquitard & 21.68 & & Y & & Y & STD \\
\hline GW-781 & GRIDE3 & Aquitard & 71.07 & & & & Y & STD \\
\hline GW-782 & GRIDE3 & Aquitard & 38.23 & & & & $Y$ & STD \\
\hline GW-783 & GRIDE3 & "Aquitard & 17.98 & & & & Y & STD" \\
\hline GW-791 & GRIDD2 & Aquitard & 72.45 & & & & Y & STD" \\
\hline GW-792 & GRIDD2 & Aquitard & 31.99 & & & & Y & STD \\
\hline GW-816 & EXP-SR & Aquitard & 17.99 & & & Y & & STD \\
\hline GW-820 & B9201-2 & Aquifer & 17.18 & & $\mathrm{Y}$ & & $\mathrm{D}$ & STD \\
\hline GW-960 & GRIDF2 & Aquitard & & & Y & & Y & STD \\
\hline GHK2.51WSW & EXP-SW & & & & & & Y & STD \\
\hline NPR12.0SW & EXP-SW & & & & & & Y & STD \\
\hline NPR23.0SW & EXP-SW & & & & & & Y & STD \\
\hline
\end{tabular}




\section{Table B.1 (continued)}

Notes:

1 BCK - Bear Creek Kilometer (surface water station)

ER - Equipment rinsate sample

FB - Field blank sample

GW - Groundwater monitoring well

GHK - Gum Hollow Kilometer (surface water station)

NPR - North of Pine Ridge (surface water station)

NT - North Tributary to Bear Creek (surface water station)

S17 - Surface water station in SCR5

SCR - South Chestnut Ridge (spring or surface water station)

SS - Spring sampling location: South Side of Bear Creek

2 B4 - Beta-4 Security Pits

B8110 - Building 81-10

B9103 - Building 9103

B9201-2 - Building 9201-2

B9201-5 - Building 9201-5

BG - Bear Creek Burial Grounds Waste Management Area

CPT - Coal Pile Trench

CRSP - Chestnut Ridge Security Pits

EXP-B - Exit Pathway Picket B

EXP-C - Exit Pathway Picket C

EXP-E - Maynardville Limestone Exit Pathway Picket E

EXP-J - Maynardville Limestone Exit Pathway Picket J

EXP-NPR - Surface water sampling station located where drainage exits the Oak Ridge Reservation, north of Pine Ridge

EXP-SR - Exit pathway well in the gap through Pine Ridge along Scarboro Road

EXP-SW - Spring or Surface Water Location

FCAP - Filled Coal Ash Pond

FTF - Fire Training Facility

GRID - Comprehensive Groundwater Monitoring Plan Grid Location

LD - Lysimeter Demonstration Site

NHP - New Hope Pond

OLF - Oil Landfarm Waste Management Area

RG - Rust Garage Area

RS - Rust Spoil Area

S2 - S-2 Site

S3 - S3 Site

SPI - Spoil Area I

SY - Y-12 Salvage Yard

T0134 - Underground Storage Tank 0134-U

WCPA - Waste Coolant Processing Area

Y12 - Y-12 Complex

3 Hydrostratigrhaphic Unit:

Aquifer - Formations of the Knox Group and the Maynardville Limestone (Conasauga Group)

Aquitard - Formations of the Conasauga Group, excluding the Maynardville Limestone

4 Tag Depth:

The distance measured (in ft) from the top of the well casing to the bottom of the well, as recorded during well inspections.

For well GW-722, the value is the depth (below the top of well casing) of the sampling port. 


\section{Table B.1 (continued)}

\section{Notes: (continued)}

5 Details regarding the monitoring frequency for each location is provided in Appendix F.

Groundwater Monitoring Schedules (Appendix C) provide the sequence for collecting samples during each quarterly sampling event and includes the waste stream identification for groundwater purged from each monitoring well. The Waste Management Plan for sampling activities is in Appendix G.

Y - Sample collection will be performed during the CY 2008 quarter

D - A field duplicate sample will collected in addition to the regular sample

* - Sample collection also with a passive diffusion bag sampler

6 Table B.2 provides a comprehensive list of analytes, analytical methods, and the associated parameter group.

STD - Standard administrative parameter group, including the following elementary parameter groups:

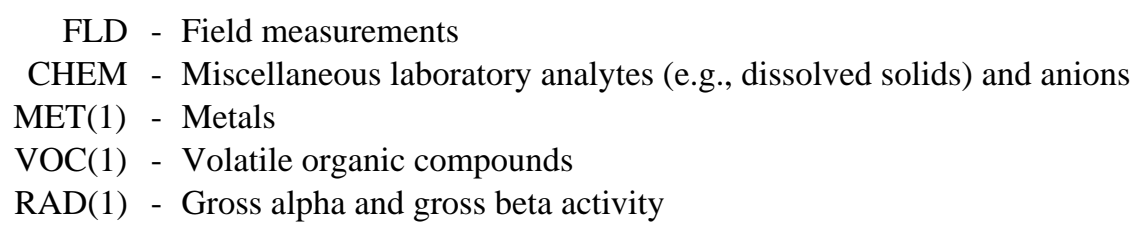

Radionuclide Elementary Parameter Groups:

RAD(3) - Uranium-234, -235, and -238

RAD(12) - Technetium-99 
B-6 
Table B.2. Field measurements and analytes that comprise the elementary parameter groups for CY 2008 groundwater and surface water samples

\begin{tabular}{|c|c|c|c|c|}
\hline $\begin{array}{c}\text { Parameter } \\
\text { Group } \\
\end{array}$ & $\begin{array}{c}\text { Measurement or } \\
\text { Analyte }\end{array}$ & $\begin{array}{c}\text { Analytical } \\
\text { Method }^{1} \\
\end{array}$ & $\begin{array}{c}\text { Reporting } \\
\text { Limit }^{2} \\
\end{array}$ & Units $^{3}$ \\
\hline \multirow[t]{6}{*}{ FLD } & Depth to Water & NA & NA & $\mathrm{ft}$ \\
\hline & Water Temperature & NA & NA & centigrade \\
\hline & $\mathrm{pH}$ & NA & NA & pH units \\
\hline & Conductivity & NA & NA & $\mu \mathrm{mho} / \mathrm{cm}$ \\
\hline & Dissolved Oxygen & NA & NA & ppm \\
\hline & Oxidation-Reduction Potential (REDOX) & NA & NA & $\mathrm{mV}$ \\
\hline \multirow{3}{*}{$\begin{array}{c}\text { CHEM } \\
\text { (miscellaneous) }\end{array}$} & Total Dissolved Solids & SM 2540C 18 & 1 & $\mathrm{mg} / \mathrm{L}$ \\
\hline & Total Suspended Solids & SM 2540D 18 & 1 & $\mathrm{mg} / \mathrm{L}$ \\
\hline & Turbidity & EPA-180.1 & 0.1 & NTU \\
\hline \multirow{6}{*}{$\begin{array}{l}\text { CHEM } \\
\text { (anions) }\end{array}$} & Bicarbonate & SM 2320B 18 & 1.0 & $\mathrm{mg} / \mathrm{L}$ \\
\hline & Carbonate & SM 2320B 18 & 1.0 & $\mathrm{mg} / \mathrm{L}$ \\
\hline & Chloride & SW846-9056 & 0.2 & $\mathrm{mg} / \mathrm{L}$ \\
\hline & Fluoride & SM 4500F 18 & 0.1 & $\mathrm{mg} / \mathrm{L}$ \\
\hline & Nitrate (as Nitrogen) & SW846-9056 & 0.028 & $\mathrm{mg} / \mathrm{L}$ \\
\hline & Sulfate & SW846-9056 & 0.25 & $\mathrm{mg} / \mathrm{L}$ \\
\hline \multirow[t]{22}{*}{ MET(1) } & Aluminum & SW846-6010B & 0.2 & $\mathrm{mg} / \mathrm{L}$ \\
\hline & Antimony & SW846-6020 & 0.0025 & $\mathrm{mg} / \mathrm{L}$ \\
\hline & Arsenic & SW846-6020 & 0.005 & $\mathrm{mg} / \mathrm{L}$ \\
\hline & Barium & SW846-6010B & 0.004 & $\mathrm{mg} / \mathrm{L}$ \\
\hline & Beryllium & SW846-6010B & 0.0005 & $\mathrm{mg} / \mathrm{L}$ \\
\hline & Boron & SW846-6010B & 0.1 & $\mathrm{mg} / \mathrm{L}$ \\
\hline & Cadmium & SW846-6020 & 0.0025 & $\mathrm{mg} / \mathrm{L}$ \\
\hline & Calcium & SW846-6010B & 0.2 & $\mathrm{mg} / \mathrm{L}$ \\
\hline & Chromium & SW846-6020 & 0.01 & $\mathrm{mg} / \mathrm{L}$ \\
\hline & Cobalt & SW846-6010B & 0.02 & $\mathrm{mg} / \mathrm{L}$ \\
\hline & Copper & SW846-6010B & 0.02 & $\mathrm{mg} / \mathrm{L}$ \\
\hline & Iron & SW846-6010B & 0.05 & $\mathrm{mg} / \mathrm{L}$ \\
\hline & Lead & SW846-6020 & 0.0005 & $\mathrm{mg} / \mathrm{L}$ \\
\hline & Lithium & SW846-6010B & 0.01 & $\mathrm{mg} / \mathrm{L}$ \\
\hline & Magnesium & SW846-6010B & 0.2 & $\mathrm{mg} / \mathrm{L}$ \\
\hline & Manganese & SW846-6010B & 0.005 & $\mathrm{mg} / \mathrm{L}$ \\
\hline & Mercury & SW846-7470 & 0.0002 & $\mathrm{mg} / \mathrm{L}$ \\
\hline & Molybdenum & SW846-6010B & 0.05 & $\mathrm{mg} / \mathrm{L}$ \\
\hline & Nickel & SW846-6020 & 0.005 & $\mathrm{mg} / \mathrm{L}$ \\
\hline & Potassium & SW846-6010B & 2 & $\mathrm{mg} / \mathrm{L}$ \\
\hline & Selenium & SW846-6020 & 0.01 & $\mathrm{mg} / \mathrm{L}$ \\
\hline & Silver & SW846-6010B & 0.02 & $\mathrm{mg} / \mathrm{L}$ \\
\hline
\end{tabular}


Table B.2 (continued)

\begin{tabular}{|c|c|c|c|c|}
\hline $\begin{array}{c}\text { Parameter } \\
\text { Group }\end{array}$ & Analyte & $\begin{array}{c}\text { Analytical } \\
\text { Method }^{1}\end{array}$ & $\begin{array}{c}\text { Reporting } \\
\text { Limit }^{2}\end{array}$ & Units $^{3}$ \\
\hline \multirow{7}{*}{$\begin{array}{c}\text { MET(1) } \\
\text { (continued) }\end{array}$} & Sodium & SW846-6010B & 0.2 & $\mathrm{mg} / \mathrm{L}$ \\
\hline & Strontium & SW846-6010B & 0.005 & $\mathrm{mg} / \mathrm{L}$ \\
\hline & Thallium & SW846-6020 & 0.0005 & $\mathrm{mg} / \mathrm{L}$ \\
\hline & Thorium & SW846-6010B & 0.2 & $\mathrm{mg} / \mathrm{L}$ \\
\hline & Uranium & SW846-6020 & 0.0005 & $\mathrm{mg} / \mathrm{L}$ \\
\hline & Vanadium & SW846-6010B & 0.02 & $\mathrm{mg} / \mathrm{L}$ \\
\hline & Zinc & SW846-6010B & 0.05 & $\mathrm{mg} / \mathrm{L}$ \\
\hline \multirow[t]{31}{*}{ VOC(1) } & Acetone & SW846-8260B-UP & 10 & $\mu \mathrm{g} / \mathrm{L}$ \\
\hline & Acrolein & SW846-8260B-UP & 10 & $\mu \mathrm{g} / \mathrm{L}$ \\
\hline & Acrylonitrile & SW846-8260B-UP & 5 & $\mu \mathrm{g} / \mathrm{L}$ \\
\hline & Benzene & SW846-8260B-UP & 5 & $\mu \mathrm{g} / \mathrm{L}$ \\
\hline & Bromochloromethane & SW846-8260B-UP & 5 & $\mu \mathrm{g} / \mathrm{L}$ \\
\hline & Bromodichloromethane & SW846-8260B-UP & 5 & $\mu \mathrm{g} / \mathrm{L}$ \\
\hline & Bromoform & SW846-8260B-UP & 5 & $\mu \mathrm{g} / \mathrm{L}$ \\
\hline & Bromomethane & SW846-8260B-UP & 5 & $\mu \mathrm{g} / \mathrm{L}$ \\
\hline & 2-Butanone & SW846-8260B-UP & 5 & $\mu \mathrm{g} / \mathrm{L}$ \\
\hline & Carbon disulfide & SW846-8260B-UP & 5 & $\mu \mathrm{g} / \mathrm{L}$ \\
\hline & Carbon tetrachloride & SW846-8260B-UP & 5 & $\mu \mathrm{g} / \mathrm{L}$ \\
\hline & Chlorobenzene & SW846-8260B-UP & 5 & $\mu \mathrm{g} / \mathrm{L}$ \\
\hline & Chloroethane & SW846-8260B-UP & 5 & $\mu \mathrm{g} / \mathrm{L}$ \\
\hline & 2-Chloroethylvinyl ether & SW846-8260B-UP & 10 & $\mu \mathrm{g} / \mathrm{L}$ \\
\hline & Chloroform & SW846-8260B-UP & 5 & $\mu \mathrm{g} / \mathrm{L}$ \\
\hline & Chloromethane & SW846-8260B-UP & 5 & $\mu \mathrm{g} / \mathrm{L}$ \\
\hline & Dibromochloromethane & SW846-8260B-UP & 5 & $\mu \mathrm{g} / \mathrm{L}$ \\
\hline & 1,2-Dibromo-3-chloropropane & SW846-8260B-UP & 10 & $\mu \mathrm{g} / \mathrm{L}$ \\
\hline & 1,2-Dibromoethane & SW846-8260B-UP & 5 & $\mu \mathrm{g} / \mathrm{L}$ \\
\hline & Dibromomethane & SW846-8260B-UP & 5 & $\mu \mathrm{g} / \mathrm{L}$ \\
\hline & 1,2-Dichlorobenzene & SW846-8260B-UP & 5 & $\mu \mathrm{g} / \mathrm{L}$ \\
\hline & 1,4-Dichlorobenzene & SW846-8260B-UP & 5 & $\mu \mathrm{g} / \mathrm{L}$ \\
\hline & 1,4-Dichloro-2-butene & SW846-8260B-UP & 5 & $\mu \mathrm{g} / \mathrm{L}$ \\
\hline & trans-1,4-Dichloro-2-butene & SW846-8260B-UP & 5 & $\mu \mathrm{g} / \mathrm{L}$ \\
\hline & Dichlorodifluoromethane & SW846-8260B-UP & 5 & $\mu \mathrm{g} / \mathrm{L}$ \\
\hline & 1,1-Dichloroethane & SW846-8260B-UP & 5 & $\mu \mathrm{g} / \mathrm{L}$ \\
\hline & 1,2-Dichloroethane & SW846-8260B-UP & 5 & $\mu \mathrm{g} / \mathrm{L}$ \\
\hline & 1,1-Dichloroethene & SW846-8260B-UP & 5 & $\mu \mathrm{g} / \mathrm{L}$ \\
\hline & cis-1,2-Dichloroethene & SW846-8260B-UP & 5 & $\mu \mathrm{g} / \mathrm{L}$ \\
\hline & trans-1,2-Dichloroethene & SW846-8260B-UP & 5 & $\mu \mathrm{g} / \mathrm{L}$ \\
\hline & 1,2-Dichloropropane & SW846-8260B-UP & 5 & $\mu g / L$ \\
\hline
\end{tabular}


Table B.2 (continued)

\begin{tabular}{|c|c|c|c|c|}
\hline $\begin{array}{l}\text { Parameter } \\
\text { Group }\end{array}$ & Analyte & $\begin{array}{c}\text { Analytical } \\
\text { Method }^{1}\end{array}$ & $\begin{array}{c}\text { Reporting } \\
\text { Limit }^{2}\end{array}$ & Units $^{3}$ \\
\hline \multirow{23}{*}{$\begin{array}{c}\text { VOC(1) } \\
\text { (continued) }\end{array}$} & cis-1,3-Dichloropropene & SW846-8260B-UP & 5 & $\mu \mathrm{g} / \mathrm{L}$ \\
\hline & trans-1,3-Dichloropropene & SW846-8260B-UP & 5 & $\mu g / L$ \\
\hline & $\begin{array}{rr}\text { Ethanol } \\
\end{array}$ & SW846-8260B-UP & 200 & $\mu \mathrm{g} / \mathrm{L}$ \\
\hline & Ethylbenzene & SW846-8260B-UP & 5 & $\mu \mathrm{g} / \mathrm{L}$ \\
\hline & Ethyl methacrylate & SW846-8260B-UP & 5 & $\mu \mathrm{g} / \mathrm{L}$ \\
\hline & 2-Hexanone & SW846-8260B-UP & 5 & $\mu \mathrm{g} / \mathrm{L}$ \\
\hline & Iodomethane & SW846-8260B-UP & 5 & $\mu \mathrm{g} / \mathrm{L}$ \\
\hline & 4-Methyl-2-pentanone & SW846-8260B-UP & 5 & $\mu \mathrm{g} / \mathrm{L}$ \\
\hline & Methylene chloride & SW846-8260B-UP & 5 & $\mu \mathrm{g} / \mathrm{L}$ \\
\hline & Styrene & SW846-8260B-UP & 5 & $\mu \mathrm{g} / \mathrm{L}$ \\
\hline & 1,1,1,2-Tetrachloroethane & SW846-8260B-UP & 5 & $\mu \mathrm{g} / \mathrm{L}$ \\
\hline & 1,1,2,2-Tetrachloroethane & SW846-8260B-UP & 5 & $\mu \mathrm{g} / \mathrm{L}$ \\
\hline & Tetrachloroethene & SW846-8260B-UP & 5 & $\mu \mathrm{g} / \mathrm{L}$ \\
\hline & Toluene & SW846-8260B-UP & 5 & $\mu \mathrm{g} / \mathrm{L}$ \\
\hline & Total Xylene & SW846-8260B-UP & 5 & $\mu \mathrm{g} / \mathrm{L}$ \\
\hline & 1,1,1-Trichloroethane & SW846-8260B-UP & 5 & $\mu \mathrm{g} / \mathrm{L}$ \\
\hline & 1,1,2-Trichloroethane & SW846-8260B-UP & 5 & $\mu \mathrm{g} / \mathrm{L}$ \\
\hline & Trichloroethene & SW846-8260B-UP & 5 & $\mu \mathrm{g} / \mathrm{L}$ \\
\hline & Trichlorofluoromethane & SW846-8260B-UP & 5 & $\mu \mathrm{g} / \mathrm{L}$ \\
\hline & 1,2,3-Trichloropropane & SW846-8260B-UP & 10 & $\mu \mathrm{g} / \mathrm{L}$ \\
\hline & 1,1,2-Trichloro-1,2,2-trifluoroethane & SW846-8260B-UP & 5 & $\mu \mathrm{g} / \mathrm{L}$ \\
\hline & Vinyl acetate & SW846-8260B-UP & 10 & $\mu \mathrm{g} / \mathrm{L}$ \\
\hline & Vinyl chloride & SW846-8260B-UP & 2 & $\mu \mathrm{g} / \mathrm{L}$ \\
\hline $\operatorname{VOC}(3)$ & Methanol & SW846-8015B & 5,000 & $\mu g / L$ \\
\hline $\mathrm{RAD}(1)$ & Gross Alpha Activity & EPA-900.0 & 5 & $\mathrm{pCi} / \mathrm{L}$ \\
\hline $\mathrm{RAD}(1)$ & Gross Beta Activity & EPA-900.0 & 10 & $\mathrm{pCi} / \mathrm{L}$ \\
\hline $\mathrm{RAD}(3)$ & Uranium-234, -235, \& -238 & Y/P65-7061 & 0.4 & $\mathrm{pCi} / \mathrm{L}$ \\
\hline $\operatorname{RAD}(12)$ & Technetium-99 & Y/P65-7060 & 15 & $\mathrm{pCi} / \mathrm{L}$ \\
\hline
\end{tabular}

Notes:

$1 \quad$ N/A - Not Applicable

Field measurements are performed in accordance with the following BWXT Management Requirements operating procedures:

\begin{tabular}{|cc|cc|}
\hline Field Measurement & Procedure & Field Measurement & Procedure \\
\hline Depth to Water & Y50-71-015 & Dissolved Oxygen & Y50-71-032 \\
Water Temperature & Y50-71-030,-014 & REDOX & Y50-71-033 \\
pH & Y50-71-031,-014 & Pressure Profile & Y50-71-019 \\
Conductivity & Y50-71-034,-022 & & \\
\hline
\end{tabular}


Table B.2 (continued)

Notes: (continued)

1 (continued)

Analytical methods from:

- EPA - Methods for Chemical Analysis of Water and Wastes

(U.S. Environmental Protection Agency 1983)

- SM - Standard Methods for the Evaluation of Water and Wastewater, $18^{\text {th }}$ Edition (American Public Health Association 1992)

- SW846 - Test Methods for Evaluating Solid Waste Physical/Chemical Methods (U.S. Environmental Protection Agency 1996)

- BWXT ACO Procedures applicable to the methods shown above in the table:

\begin{tabular}{|cl|cl|}
\hline Method & ACO Procedure & Method & \multicolumn{1}{c|}{ ACO Procedure } \\
\hline EPA-180.1 & Y/P65-7615 & SW846-6010B & Y50-AC-65-0040 \\
EPA-900.0 & Y50-AC-65-7074 & SW846-6020 & Y/P65-0034 \\
& & SW846-7470 & Y50-AC-65-7470 \\
SM 2320B 18 & Y/P65-7639 & SW846-8015B & Y/P65-7348 \\
SM 2540C 18 & Y-50-AC-65-7914 & SW846-8260B-UP & Y/P65-SW846-8260B \\
SM 2540D 18 & Y/P65-7918 & SW846-9056 & Y50-AC-65-7619 \\
SM 4500F 18 & Y/P65-7602 & Y/P65-7060 & Y/P65-7060 \\
& & Y/P65-7061 & Y/P65-7061 \\
\hline
\end{tabular}

NA - not applicable

$\operatorname{VOC}(1,3) \quad$ - Reporting limits are contract-required quantitation limits; also report estimated values (with qualifier) below this limit and above the method detection limit.

RAD $(1,3,12)$ - Reporting limits are target minimum detectable activities (MDAs) that may be obtained under optimal analytical conditions; actual MDAs are sample-specific and may vary significantly from the target value.

3

$\mathrm{ft}$ - feet

$\mu \mathrm{g} / \mathrm{L} \quad$ - micrograms per liter

$\mu \mathrm{mho} / \mathrm{cm}$ - micromhos per centimeter

$\mathrm{mg} / \mathrm{L} \quad$ - milligrams per liter

$\mathrm{mV}$ - millivolts

NTU - nephelometric turbidity units

ppm - parts per million

pCi/L - picoCuries per liter 
APPENDIX C

CY 2008 GROUNDWATER MONITORING SCHEDULES (Insert When Issued, Before Each Quarterly Sampling Event) 
APPENDIX D

ADDENDA TO THE CY 2008 SAMPLING AND ANALYSIS PLAN (if issued) 
APPENDIX E

LABORATORY REQUIREMENTS

(Bottle Lists, Holding Times, Turnaround Time, Elevated Minimum Activity) 


\section{STD}

\begin{tabular}{||l|c|c|l||}
\hline \multicolumn{1}{|c|}{ Parameter } & Lab Tests & $\begin{array}{c}\text { Chemical } \\
\text { Preservative }\end{array}$ & \multicolumn{1}{|c||}{ Bottle Types/Size } \\
\hline \hline $\begin{array}{l}\text { Anions, Turbidity, } \\
\text { Fluoride, Carbonate and } \\
\text { Bicarbonate }\end{array}$ & $\begin{array}{c}\text { ANIONS, } \\
\text { TURBIDITY, } \\
\text { FLUORIDE, } \\
\text { ALKALINITY-I }\end{array}$ & None & $1-250 \mathrm{~mL}$ polyethylene \\
\hline Total Suspended Solids & SOLIDS-TOT-S & None & $1-250 \mathrm{~mL}$ polyethylene \\
\hline Total Dissolved Solids & SOLIDS-TOT-D & None & $1-250 \mathrm{~mL}$ polyethylene \\
\hline $\begin{array}{l}\text { Total Metals (ICP,ICP- } \\
\text { MS, and Hg) }\end{array}$ & $\begin{array}{c}\text { ICP6010, } \\
\text { ICPMSGW, HG7470 }\end{array}$ & $\mathrm{HNO}_{3}$ & $1-500 \mathrm{~mL}$ polyethylene \\
\hline Radiochemistry (UV) & GROSSAB-ENV & $\mathrm{HNO}_{3}$ & $1-1 \mathrm{~L}$ polyethylene \\
\hline Volatiles & VOA8260GW & None & $\begin{array}{l}2-40 \mathrm{~mL} \text { amber glass } \\
\text { with Teflon lined septum } \\
\text { lids }\end{array}$ \\
\hline $\begin{array}{l}\text { Trip Blank (VOA) } \\
\text { (one per cooler) }\end{array}$ & VOA8260GW & None & $\begin{array}{l}1-40 \mathrm{~mL} \text { amber glass } \\
\text { with Teflon lined septum } \\
\text { lid }\end{array}$ \\
\hline
\end{tabular}

STD:

LIMS LAB TEST ID

CHEM ALKALINITY-I, ANIONS, FLUORIDE, SOLIDS-TOT-S, SOLIDS-TOT-D, TURBIDITY

MET(1) ICP6010, ICPMSGW and HG7470

VOC(1) VOA8260GW

RAD(1) Gross Alpha Beta (GROSSAB-ENV)

${ }^{1}$ Samples chilled to $4+/-2 \mathrm{C}$ 


\section{STD, RAD $(3,12)$}

\begin{tabular}{||l|c|c|l||}
\hline \multicolumn{1}{|c|}{ Parameter } & Lab Tests & $\begin{array}{c}\text { Chemical } \\
\text { Preservative }\end{array}$ & \multicolumn{1}{|c||}{ Bottle Types/Size } \\
\hline \hline $\begin{array}{l}\text { Anions, Turbidity, } \\
\text { Fluoride, Carbonate and } \\
\text { Bicarbonate }\end{array}$ & $\begin{array}{c}\text { ANIONS, } \\
\text { TURBIDITY, } \\
\text { FLUORIDE, } \\
\text { ALKALINITY-I }\end{array}$ & None & $1-250 \mathrm{~mL}$ polyethylene \\
\hline Total Suspended Solids & SOLIDS-TOT-S & None & $1-250 \mathrm{~mL}$ polyethylene \\
\hline Total Dissolved Solids & SOLIDS-TOT-D & None & $1-250 \mathrm{~mL}$ polyethylene \\
\hline $\begin{array}{l}\text { Total Metals (ICP,ICP- } \\
\text { MS, and Hg) }\end{array}$ & $\begin{array}{c}\text { ICP6010, } \\
\text { ICPMSGW, HG7470 }\end{array}$ & $\mathrm{HNO}_{3}$ & $1-500 \mathrm{~mL}$ polyethylene \\
\hline $\begin{array}{l}\text { Gross Alpha/Beta, TC-99 } \\
\text { and Uranium-234, 235, - } \\
\text { 238 }\end{array}$ & $\begin{array}{c}\text { GROSSAB-ENV } \\
\text { TC99-ENV } \\
\text { ASPECU-ENV }\end{array}$ & $\mathrm{HNO}_{3}$ & $\begin{array}{l}1-1 \mathrm{~L} \text { polyethylene \& } \\
1-500-\mathrm{mL} \text { polyethylene }\end{array}$ \\
\hline Volatiles & VOA8260GW & None & $\begin{array}{l}2-40 \mathrm{~mL} \text { amber glass } \\
\text { with Teflon lined septum } \\
\text { lids }\end{array}$ \\
\hline $\begin{array}{l}\text { Trip Blank (VOA) } \\
\text { one per cooler) }\end{array}$ & VOA8260GW & None & $\begin{array}{l}1-40 \mathrm{~mL} \text { amber glass } \\
\text { with Teflon lined septum } \\
\text { lid }\end{array}$ \\
\hline
\end{tabular}

\section{STD:}

CHEM

LIMS LAB TEST ID

$\begin{array}{ll}\text { MET(1) } & \text { SOLIDS-TOT-D, TURBIDITY } \\ \text { ICP6010, ICPMSGW and HG7470 }\end{array}$

VOC(1) VOA8260GW

RAD(1) Gross Alpha Beta (GROSSAB-ENV)

$\operatorname{RAD}(3,12) \quad T C-99 L S-E N V$, and ASPECU-ENV

${ }^{1}$ Samples chilled to $4+/-2 \mathrm{C}$ 


\section{STD, RAD (12)}

\begin{tabular}{|c|c|c|c|}
\hline Parameter & Lab Tests & $\begin{array}{c}\text { Chemical }^{-} \\
\text {Preservative }^{1}\end{array}$ & Bottle Types/Size \\
\hline $\begin{array}{l}\text { Anions, Turbidity, } \\
\text { Fluoride, Carbonate and } \\
\text { Bicarbonate }\end{array}$ & $\begin{array}{l}\text { ANIONS, } \\
\text { TURBIDITY, } \\
\text { FLUORIDE, } \\
\text { ALKALINITY-I }\end{array}$ & None & 1 - $250 \mathrm{~mL}$ polyethylene \\
\hline Total Suspended Solids & SOLIDS-TOT-S & None & 1 - $250 \mathrm{~mL}$ polyethylene \\
\hline Total Dissolved Solids & SOLIDS-TOT-D & None & 1 - $250 \mathrm{~mL}$ polyethylene \\
\hline $\begin{array}{l}\text { Total Metals (ICP,ICP- } \\
\text { MS, and Hg) }\end{array}$ & $\begin{array}{c}\text { ICP6010, } \\
\text { ICPMSGW, HG7470 }\end{array}$ & $\mathrm{HNO}_{3}$ & 1 - $500 \mathrm{~mL}$ polyethylene \\
\hline $\begin{array}{l}\text { Gross Alpha/Beta and } \\
\text { Tc- } 99\end{array}$ & $\begin{array}{c}\text { GROSSAB-ENV } \\
\text { TC99-ENV }\end{array}$ & $\mathrm{HNO}_{3}$ & 1- 1 L polyethylene \& \\
\hline Volatiles & VOA8260GW & None & $\begin{array}{l}2-40 \mathrm{~mL} \text { amber glass } \\
\text { with Teflon lined septum } \\
\text { lids }\end{array}$ \\
\hline $\begin{array}{l}\text { Trip Blank (VOA) } \\
\text { (one per cooler) }\end{array}$ & VOA8260GW & None & $\begin{array}{l}1-40 \mathrm{~mL} \text { amber glass } \\
\text { with Teflon lined septum } \\
\text { lid }\end{array}$ \\
\hline
\end{tabular}

STD:

LIMS LAB TEST ID

CHEM ALKALINITY-I, ANIONS, FLUORIDE, SOLIDS-TOT-S, SOLIDS-TOT-D, TURBIDITY

MET(1) ICP6010, ICPMSGW and HG7470

VOC(1) VOA8260GW

RAD(1) Gross Alpha Beta (GROSSAB-ENV)

RAD(12) TC-99LS-ENV

${ }^{1}$ Samples chilled to $4+/-2 \mathrm{C}$ 


\section{STD,VOC (3)}

\begin{tabular}{||l|c|c|l||}
\hline \multicolumn{1}{|c|}{ Parameter } & Lab Tests & $\begin{array}{c}\text { Chemical } \\
\text { Preservative }\end{array}$ & \multicolumn{1}{|c||}{ Bottle Types/Size } \\
\hline \hline $\begin{array}{l}\text { Anions, Turbidity, } \\
\text { Fluoride, Carbonate and } \\
\text { Bicarbonate }\end{array}$ & $\begin{array}{c}\text { ANIONS, } \\
\text { TURBIDITY, } \\
\text { FLUORIDE, } \\
\text { ALKALINITY-I }\end{array}$ & None & $1-250 \mathrm{~mL}$ polyethylene \\
\hline Total Suspended Solids & SOLIDS-TOT-S & None & $1-250 \mathrm{~mL}$ polyethylene \\
\hline Total Dissolved Solids & SOLIDS-TOT-D & None & $1-250 \mathrm{~mL}$ polyethylene \\
\hline $\begin{array}{l}\text { Total Metals (ICP,ICP- } \\
\text { MS, and Hg) }\end{array}$ & $\begin{array}{c}\text { ICP6010, } \\
\text { ICPMSGW, HG7470 }\end{array}$ & $\mathrm{HNO}_{3}$ & $1-500 \mathrm{~mL}$ polyethylene \\
\hline Radiochemistry (UV) & GROSSAB-ENV & $\mathrm{HNO}_{3}$ & $1-1 \mathrm{~L}$ polyethylene \\
\hline Volatiles & VOA8260GW & None & $\begin{array}{l}2-40 \mathrm{~mL} \text { amber glass } \\
\text { with Teflon lined septum } \\
\text { lids }\end{array}$ \\
\hline Methanol & NHO8015 & None & $\begin{array}{l}2-40 \mathrm{~mL} \text { amber glass } \\
\text { with Teflon lined septum } \\
\text { lids }\end{array}$ \\
\hline \hline $\begin{array}{l}\text { Trip Blank (VOA) } \\
\text { (one per cooler) }\end{array}$ & VOA8260GW & None & $\begin{array}{l}1-40 \mathrm{~mL} \text { amber glass } \\
\text { with Teflon lined septum } \\
\text { lid }\end{array}$ \\
\hline
\end{tabular}

\section{STD: $\quad$ LIMS LAB TEST ID}

CHEM ALKALINITY-I, ANIONS, FLUORIDE, SOLIDS-TOT-S,

$\begin{array}{ll}\text { MET(1) } & \text { SOLIDS-TOT-D, TURBIDITY } \\ \text { ICP6010, ICPMSGW and HG7470 }\end{array}$

VOC(1) VOA8260GW

RAD(1)Gross Alpha Beta (GROSSAB-ENV)

METHANOL NHO8015

\footnotetext{
${ }^{1}$ Samples chilled to $4+/-2 \mathrm{C}$ 


\section{WESTBAY}

\begin{tabular}{||l|c|c|l||}
\hline \multicolumn{1}{|c|}{ Parameter } & Lab Tests & $\begin{array}{c}\text { Chemical } \\
\text { Preservative }\end{array}$ & \multicolumn{1}{|c||}{ Bottle Types/Size } \\
\hline \hline $\begin{array}{l}\text { Anions, Turbidity, } \\
\text { Fluoride, Carbonate and } \\
\text { Bicarbonate }\end{array}$ & $\begin{array}{c}\text { ANIONS, } \\
\text { TURBIDITY, } \\
\text { FLUORIDE, } \\
\text { ALKALINITY-I }\end{array}$ & None & $1-250 \mathrm{~mL}$ polyethylene \\
\hline Total Suspended Solids & SOLIDS-TOT-S & None & $1-250 \mathrm{~mL}$ polyethylene \\
\hline Total Dissolved Solids & SOLIDS-TOT-D & None & $1-250 \mathrm{~mL}$ polyethylene \\
\hline $\begin{array}{l}\text { Total Metals (ICP,ICP- } \\
\text { MS, and Hg) }\end{array}$ & $\begin{array}{c}\text { ICP6010, } \\
\text { ICPMSGW, HG7470 }\end{array}$ & $\mathrm{HNO}_{3}$ & $1-250 \mathrm{~mL}$ polyethylene \\
\hline Radiochemistry (UV) & GROSSAB-ENV & $\mathrm{HNO}_{3}$ & $\begin{array}{l}1-500 \mathrm{~mL} \\
\text { polyethylene }\end{array}$ \\
\hline Volatiles & VOA8260GW & None & $\begin{array}{l}2-40 \mathrm{~mL} \text { amber glass } \\
\text { with Teflon lined septum } \\
\text { lids }\end{array}$ \\
\hline $\begin{array}{l}\text { Trip Blank (VOA) } \\
\text { (one per cooler) }\end{array}$ & VOA8260GW & None & $\begin{array}{l}1-40 \mathrm{~mL} \text { amber glass } \\
\text { with Teflon lined septum } \\
\text { lid }\end{array}$ \\
\hline \hline
\end{tabular}

STD:

LIMS LAB TEST ID

CHEM ALKALINITY-I, ANIONS, FLUORIDE, SOLIDS-TOT-S, SOLIDS-TOT-D, TURBIDITY

MET(1) ICP6010, ICPMSGW and HG7470

VOC(1) VOA8260GW

RAD(1) Gross Alpha Beta (GROSSAB-ENV)

${ }^{1}$ Samples chilled to $4+/-2 \mathrm{C}$ 


\section{$\operatorname{VOC}(1)$}

\begin{tabular}{||c|c|c|c||}
\hline \multicolumn{1}{|c|}{ Parameter } & Lab Tests & $\begin{array}{c}\text { Chemical } \\
\text { Preservative }\end{array}$ & Bottle Types/Size \\
\hline \hline Volatiles & VOA8260GW & None & $\begin{array}{l}2-40 \mathrm{~mL} \text { amber glass } \\
\text { with Teflon lined septum } \\
\text { lids }\end{array}$ \\
\hline
\end{tabular}

STD:

VOC(1) VOA8260GW

LIMS LAB TEST ID

${ }^{1}$ Samples chilled to $4+/-2 \mathrm{C}$ 


\section{ESTABLISHED HOLDING TIMES}

\begin{tabular}{|l|l|}
\hline Parameter & Holding Times \\
\hline Alkalinity (Carbonate, Bicarbonate) & 14 days \\
\hline Anions (Chloride, Nitrate, Sulfate) & $48 \mathrm{hr}$ \\
\hline Fluoride & 28 days \\
\hline Mercury & 28 days \\
\hline Metals (ICP, ICPMS) & 6 months \\
\hline Radiochemistry (except tritium) & 6 months \\
\hline Solids, Total Dissolved & 7 days \\
\hline Solids, Total Suspended & 7 days \\
\hline Tritium & No EPA guidance \\
\hline VOA & 7 days \\
\hline
\end{tabular}

\section{ESTABLISHED TURNAROUND TIMES}

The Groundwater Protection Program and the Analytical Chemistry Organization (ACO) laboratory have agreed upon a turnaround time, such that the analytical data generated from each sampling location will be completed within 35 days of receipt. Every two weeks, data that has been approved since the previous twoweek period will be transmitted in the form of hard copy of the approved lab reports for each location, along with an electronic copy in a standardized and compatible format (please see the most recent version of the Y-12 Plant Groundwater Protection Program Data Management Plan).

\section{ELEVATED MINIMUM DETECTABLE ACTIVITY}

Groundwater samples with high TDS (>1,000 mg/L) typically have elevated minimum detectable activities (MDAs) for gross alpha ( $>15 \mathrm{pCi} / \mathrm{L}$ ) and gross beta (> $50 \mathrm{pCi} / \mathrm{L})$. However, the MDAs for specific isotopic analyses are unaffected by the sample solid content. For samples with gross activity results that are less than an elevated MDA, and specific isotopic analyses have not been requested, the laboratory will issue a request to analyze for the principal alpha- or beta-emitting isotopes. That is, if the gross alpha MDA exceeds $15 \mathrm{pCi} / \mathrm{L}$ and the result is less than $15 \mathrm{pCi} / \mathrm{L}$, then the laboratory will request analyses of isotopic uranium (by method Y/P65-7061). Similarly, if a sample has an elevated gross beta MDA (>50 pCi/L) and the result is less than the MDA, then the laboratory would request analysis of technetium-99 activity. These requests will be approved by the Y-12 Groundwater Protection Program manager, or designee, before analyses are performed. 
APPENDIX F

CY 2008 SAMPLING FREQUENCY FOR MONITORING WELLS 


\section{Appendix F. CY 2008 Sampling Frequency for Monitoring Wells}

\begin{tabular}{|c|c|c|c|c|c|}
\hline \multirow{2}{*}{$\begin{array}{l}\text { Monitoring } \\
\text { Well }\end{array}$} & \multirow{2}{*}{ Regime $^{1}$} & \multicolumn{2}{|c|}{ Sampling Frequency ${ }^{2}$} & \multirow{2}{*}{$\begin{array}{c}\text { No. of Samples }{ }^{3} \\
1996-2007\end{array}$} & \multirow{2}{*}{$\begin{array}{c}\text { Explanation }^{4} \\
\text { (CY } 2008 \text { differs from MOP) }\end{array}$} \\
\hline & & GWPP MOP & CY 2008 & & \\
\hline $55-1 \mathrm{~A}$ & EF & Annual & Annual & 5 & \\
\hline $55-2 A$ & EF & Semiannual & Annual & 4 & GWMR \\
\hline $55-2 B$ & EF & Semiannual & Annual & 9 & GWMR \\
\hline $55-2 C$ & EF & Annual & Annual & 11 & \\
\hline $55-3 A$ & EF & Semiannual & Annual & 5 & GWMR \\
\hline $55-3 B$ & $E F$ & Semiannual & Annual & 5 & GWMR \\
\hline $55-3 C$ & EF & Semiannual & Annual & 6 & GWMR \\
\hline $55-6 \mathrm{~A}$ & EF & TBD & Even & 3 & Selected for Review \\
\hline $56-1 \mathrm{~A}$ & EF & Semiannual & Annual & 4 & GWMR \\
\hline $56-1 C$ & EF & TBD & Annual & 1 & Selected for Review \\
\hline $56-2 A$ & EF & Annual & Annual & 6 & \\
\hline $56-2 B$ & EF & Annual & Annual & 6 & \\
\hline $56-2 C$ & EF & Annual & Annual & 8 & \\
\hline $56-3 A$ & EF & Semiannual & Annual & 4 & GWMR \\
\hline $56-3 B$ & EF & Semiannual & Annual & 4 & GWMR \\
\hline $56-3 C$ & EF & Semiannual & Annual & 5 & GWMR \\
\hline $56-4 \mathrm{~A}$ & EF & Semiannual & Annual & 4 & GWMR \\
\hline $56-6 \mathrm{~A}$ & $E F$ & Semiannual & Annual & 4 & GWMR \\
\hline $56-8 \mathrm{~A}$ & EF & Semiannual & Annual & 4 & GWMR \\
\hline $60-1 \mathrm{~A}$ & EF & Semiannual & Annual & 4 & GWMR \\
\hline GW-006 & $\mathrm{BC}$ & TBD & Even & 8 & Selected for Review \\
\hline GW-014 & $\mathrm{BC}$ & Semiannual & Annual & 8 & GWMR \\
\hline GW-058 & $\mathrm{BC}$ & Even & Even & 1 & \\
\hline GW-065 & $\mathrm{BC}$ & TBD & SemiAnnual & 0 & Selected for Review \\
\hline GW-068 & $\mathrm{BC}$ & Even & Even & 3 & \\
\hline GW-071 & $\mathrm{BC}$ & Semiannual & SemiAnnual & 10 & \\
\hline GW-082 & $\mathrm{BC}$ & Annual & Annual & 19 & \\
\hline GW-085 & $\mathrm{BC}$ & Semiannual & SemiAnnual & 24 & \\
\hline GW-089 & $\mathrm{BC}$ & Odd & Annual & 1 & MAROS \\
\hline GW-098 & $\mathrm{BC}$ & Annual & Annual & 8 & \\
\hline GW-105 & EF & Annual & Annual & 4 & \\
\hline GW-106 & EF & Annual & Annual & 4 & \\
\hline GW-109 & EF & Annual & Annual & 8 & \\
\hline GW-122 & $\mathrm{BC}$ & Annual & Annual & 4 & \\
\hline GW-148 & EF & Even & Even & 11 & \\
\hline GW-153 & EF & Annual & Annual & 24 & \\
\hline GW-173 & $\mathrm{CR}$ & TBD & Even & 2 & Selected for Review \\
\hline GW-175 & $\mathrm{CR}$ & Even & Even & 6 & \\
\hline GW-178 & $\mathrm{CR}$ & Even & Even & 3 & \\
\hline GW-190 & EF & TBD & Even & 2 & Selected for Review \\
\hline GW-192 & EF & Annual & Annual & 16 & \\
\hline GW-204 & EF & Annual & Annual & 19 & \\
\hline GW-220 & EF & Semiannual & SemiAnnual & 31 & \\
\hline GW-225 & $\mathrm{BC}$ & Semiannual & SemiAnnual & 16 & \\
\hline GW-226 & $\mathrm{BC}$ & Semiannual & SemiAnnual & 22 & \\
\hline GW-227 & $\mathrm{BC}$ & Even & Even & 2 & \\
\hline GW-229 & $\mathrm{BC}$ & Annual & Annual & 12 & \\
\hline
\end{tabular}




\section{Appendix F. CY 2008 Sampling Frequency for Monitoring Wells}

\begin{tabular}{|c|c|c|c|c|c|}
\hline \multirow{2}{*}{$\begin{array}{l}\text { Monitoring } \\
\text { Well }\end{array}$} & \multirow{2}{*}{ Regime $^{1}$} & \multicolumn{2}{|c|}{ Sampling Frequency ${ }^{2}$} & \multirow{2}{*}{$\begin{array}{c}\text { No. of Samples } \\
\text { 1996-2007 }\end{array}$} & \multirow{2}{*}{$\begin{array}{c}\text { Explanation }^{4} \\
\text { (CY } 2008 \text { differs from MOP) }\end{array}$} \\
\hline & & GWPP MOP & CY 2008 & & \\
\hline GW-240 & $\mathrm{EF}$ & Annual & Annual & 10 & \\
\hline GW-246 & $\mathrm{BC}$ & Semiannual & SemiAnnual & 6 & \\
\hline GW-251 & $\mathrm{EF}$ & Annual & Annual & 25 & \\
\hline GW-257 & $\mathrm{BC}$ & Annual & Annual & 4 & \\
\hline GW-259 & $\mathrm{BC}$ & Even & SemiAnnual & 0 & MAROS \\
\hline GW-265 & $\mathrm{EF}$ & Semiannual & Annual & 5 & GWMR \\
\hline GW-269 & $\mathrm{EF}$ & Semiannual & Annual & 7 & GWMR \\
\hline GW-274 & $\mathrm{EF}$ & Annual & Annual & 7 & \\
\hline GW-275 & $\mathrm{EF}$ & Annual & Annual & 6 & \\
\hline GW-289 & $\mathrm{BC}$ & Annual & Annual & 8 & \\
\hline GW-306 & $\mathrm{BC}$ & TBD & SemiAnnual & 0 & Selected for Review \\
\hline GW-309 & $\mathrm{BC}$ & Even & SemiAnnual & 0 & MAROS \\
\hline GW-310 & $\mathrm{BC}$ & Even & Even & 3 & \\
\hline GW-312 & $\mathrm{BC}$ & Even & Even & 2 & \\
\hline GW-314 & $\mathrm{BC}$ & TBD & SemiAnnual & 0 & Selected for Review \\
\hline GW-315 & $\mathrm{BC}$ & Annual & Annual & 22 & \\
\hline GW-322 & $\mathrm{CR}$ & Even & Even & 5 & \\
\hline GW-332 & $\mathrm{EF}$ & Annual & Annual & 5 & \\
\hline GW-336 & $\mathrm{EF}$ & Annual & Annual & 5 & \\
\hline GW-337 & $\mathrm{EF}$ & Annual & Annual & 9 & \\
\hline GW-365 & $\mathrm{BC}$ & Even & Even & 4 & \\
\hline GW-367 & $\mathrm{BC}$ & Even & SemiAnnual & 0 & MAROS \\
\hline GW-369 & $\mathrm{BC}$ & Odd & Annual & 1 & MAROS \\
\hline GW-381 & $\mathrm{EF}$ & Annual & Annual & 15 & \\
\hline GW-383 & $\mathrm{EF}$ & Semiannual & Annual & 27 & GWMR \\
\hline GW-508 & $\mathrm{EF}$ & TBD & SemiAnnual & 0 & Selected for Review \\
\hline GW-514 & $\mathrm{CR}$ & Even & Even & 4 & \\
\hline GW-531 & $\mathrm{BC}$ & TBD & Annual & 0 & Selected for Review \\
\hline GW-537 & $\mathrm{BC}$ & Annual & Annual & 25 & \\
\hline GW-608 & $\mathrm{CR}$ & TBD & Even & 7 & Selected for Review \\
\hline GW-609 & $\mathrm{CR}$ & Even & Even & 16 & \\
\hline GW-612 & $\mathrm{CR}$ & Annual & Annual & 9 & \\
\hline GW-615 & $\mathrm{BC}$ & Annual & Annual & 7 & \\
\hline GW-616 & $\mathrm{BC}$ & Annual & Annual & 6 & \\
\hline GW-617 & $\mathrm{EF}$ & Even & Even & 6 & \\
\hline GW-619 & $\mathrm{EF}$ & Even & Even & 6 & \\
\hline GW-620 & $\mathrm{EF}$ & Annual & Annual & 25 & \\
\hline GW-623 & $\mathrm{BC}$ & TBD & SemiAnnual & 0 & Selected for Review \\
\hline GW-626 & $\mathrm{BC}$ & Semiannual & SemiAnnual & 12 & \\
\hline GW-627 & $\mathrm{BC}$ & Semiannual & SemiAnnual & 28 & \\
\hline GW-629 & $\mathrm{BC}$ & Even & Annual & 6 & MAROS \\
\hline GW-633 & $\mathrm{EF}$ & Even & Even & 14 & \\
\hline GW-648 & $\mathrm{BC}$ & TBD & Annual & 0 & Selected for Review \\
\hline GW-653 & $\mathrm{BC}$ & Annual & Annual & 24 & \\
\hline GW-656 & $\mathrm{EF}$ & Annual & Annual & 7 & \\
\hline GW-686 & $\mathrm{EF}$ & Semiannual & Annual & 6 & GWMR \\
\hline
\end{tabular}




\section{Appendix F. CY 2008 Sampling Frequency for Monitoring Wells}

\begin{tabular}{|c|c|c|c|c|c|}
\hline \multirow{2}{*}{$\begin{array}{l}\text { Monitoring } \\
\text { Well }\end{array}$} & \multirow{2}{*}{ Regime $^{1}$} & \multicolumn{2}{|c|}{ Sampling Frequency ${ }^{2}$} & \multirow{2}{*}{$\begin{array}{c}\text { No. of Samples } \\
\text { 1996-2007 }\end{array}$} & \multirow{2}{*}{$\begin{array}{c}\text { Explanation }^{4} \\
\text { (CY } 2008 \text { differs from MOP) }\end{array}$} \\
\hline & & GWPP MOP & CY 2008 & & \\
\hline GW-690 & EF & Annual & Annual & 7 & \\
\hline GW-691 & EF & Annual & Annual & 5 & \\
\hline GW-692 & EF & Annual & Annual & 6 & \\
\hline GW-694 & $\mathrm{BC}$ & Annual & Annual & 10 & \\
\hline GW-698 & EF & Semiannual & SemiAnnual & 20 & \\
\hline GW-700 & EF & Annual & Annual & 7 & \\
\hline GW-703 & $\mathrm{BC}$ & Annual & Annual & 22 & \\
\hline GW-722-14 & EF & Annual & Annual & 39 & \\
\hline GW-722-17 & EF & Annual & Annual & 39 & \\
\hline GW-722-20 & EF & Annual & Annual & 39 & \\
\hline GW-722-22 & EF & Annual & Annual & 39 & \\
\hline GW-722-33 & EF & Annual & Annual & 39 & \\
\hline GW-724 & $\mathrm{BC}$ & Annual & Annual & 22 & \\
\hline GW-725 & $\mathrm{BC}$ & Annual & Annual & 24 & \\
\hline GW-738 & $\mathrm{BC}$ & Annual & Annual & 23 & \\
\hline GW-740 & $\mathrm{BC}$ & Annual & Annual & 23 & \\
\hline GW-744 & $E F$ & Annual & Annual & 24 & \\
\hline GW-747 & EF & Annual & Annual & 24 & \\
\hline GW-748 & EF & Even & Even & 7 & \\
\hline GW-763 & EF & Annual & Annual & 27 & \\
\hline GW-769 & EF & Semiannual & SemiAnnual & 25 & \\
\hline GW-770 & EF & Semiannual & SemiAnnual & 25 & \\
\hline GW-781 & EF & Annual & Annual & 11 & \\
\hline GW-782 & EF & Annual & Annual & 26 & \\
\hline GW-783 & EF & Annual & Annual & 13 & \\
\hline GW-791 & EF & Annual & Annual & 25 & \\
\hline GW-792 & EF & Annual & Annual & 11 & \\
\hline GW-816 & EF & Annual & Annual & 24 & \\
\hline GW-820 & $E F$ & Semiannual & SemiAnnual & 10 & \\
\hline GW-829 & $\mathrm{BC}$ & Even & Even & 13 & \\
\hline GW-960 & EF & TBD & SemiAnnual & 2 & New Location \\
\hline
\end{tabular}




\title{
Appendix F. CY 2008 Sampling Frequency for Monitorng Wells
}

Notes:

1. Regime

\author{
$\mathrm{BC}=$ Bear Creek Hydrogeologic Regime \\ $\mathrm{CR}=$ Chestnut Ridge Hydrogeologic Regime \\ $\mathrm{EF}=$ Upper East Fork Poplar Creek Hydrogeologic Regime
}

2. Sampling frequency

GWPP MOP = as specified in the Y-12 Groundwater Protection Program Monitoring Optimization Plan (BWXT 2006a)

CY $2008=$ the updated sampling frequency for use beginning in CY 2008

Annual $=$ Sample collection once per year

Even $=$ Sample collection every other year, starting in 2006

Odd $=$ Sample collection every other year, starting in 2007

Semiannual $=$ Sample collection twice per year

3. Number of Samples collected from 1996-2007

The number of data points available for a MAROS assessment, based on the earliest date (January 1996) for data that was used in the initial assessment (BWXT 2005).

4. Explanation for sampling locations where the updated sampling frequency differs from the MOP sampling frequency. These changes are primarily based on the number of samples that would be included in a subsequent MAROS assessment (collected since January 1996).

GWPP = frequency reduced recommended in the CY 2006 Groundwater Monitoring Report (BWXT 2007)

MAROS = frequency increased to obtain the minimum number of samples (4 data points) needed within the subsequent 3-year (CY 2008-2010) period to perform the next MAROS assessment. 
APPENDIX G

MANAGEMENT OF PURGED GROUNDWATER 
APPENDIX G.1

\section{WASTE MANAGEMENT PLAN}




\title{
WASTE MANAGEMENT PLAN \\ for \\ Waste Streams generated from \\ Y-12 Groundwater Protection Program \\ Sampling Activities
}

\author{
Date Issued - 12/18/06 \\ prepared by: \\ Y-12 Groundwater Protection Program \\ Environmental Compliance Department \\ Y-12 National Security Complex \\ P.O. Box 2009 \\ Oak Ridge, TN 37831 \\ managed by: \\ BWXT Y-12, LLC \\ for the: \\ U.S. Department of Energy \\ Under Contract Number: \\ DE-AC05-00OR22800 \\ This document has been reviewed by a Y-12 DC/ \\ UCNI RO and has been determined to be \\ UNCLASSIFIED and contains no UCNI. This \\ review does not constitute clearance for Public \\ Release. \\ Name: L. W. McMahon [signature on file] \\ Date: \\ $12 / 18 / 06$
}




\section{Approvals}

Don Bohrman [signature on file]

Don Bohrman

$12 / 18 / 06$

Date

BWXT Y-12, LLC

Environmental Officer

Mary Wiginton [signature on file]

Mary Wiginton

$12 / 18 / 06$

BWXT Y-12, LLC

Waste Engineer

Date

Tom Conrad for D. McCune [signature on file]

12/18/06

Dave McCune

Date

Bechtel Jacobs, LLC

Waste Treatment Operations

Mark Burris [signature on file]

$12 / 18 / 06$

Brad E Skaggs or Mark S. Burris

Date

BWXT Y-12, LLC

Environmental Compliance 
Waste Management Plan for Y-12 Groundwater Protection Program Sampling Activities

\begin{tabular}{|l|l|l|l|l|}
\hline \multicolumn{1}{|c|}{ Waste stream } & \multicolumn{1}{|c|}{ Characterization ${ }^{3}$} & Segregation Requirements & \multicolumn{1}{c|}{ Packaging } & \multicolumn{1}{c|}{ Disposal Path } \\
\hline $\begin{array}{l}\text { Purge water }{ }^{2} \text { that is not } \\
\text { contained }\end{array}$ & $\begin{array}{l}\text { Non-hazardous, non-radiological } \\
\text { contaminated waters. Analytical results } \\
\text { indicate constituents in the water are less } \\
\text { than Safe Drinking Water Act Maximum } \\
\text { Contaminant Levels (MCL). In } \\
\text { addition, historical knowledge of } \\
\text { relevant groundwater plumes at the Y-12 } \\
\text { National Security Complex confirm the } \\
\text { non-detection of contaminants, or the } \\
\text { detection of contaminants (J values), but } \\
\text { still below the MCL. See the most } \\
\text { current GWPP Groundwater Monitoring } \\
\text { Data Compendium }\end{array}$ & Not contained & $\begin{array}{l}\text { ACO technicians will } \\
\text { dispense/dispose of waters directly } \\
\text { to ground surface at the well } \\
\text { location. }\end{array}$ & \\
\hline
\end{tabular}




\begin{tabular}{|c|c|c|c|c|}
\hline Waste stream & Characterization $^{3}$ & Segregation Requirements & Packaging & Disposal Path \\
\hline $\begin{array}{l}\text { SID }^{1} 2212 \text { purge water } \\
\text { (non-regulated and non- } \\
\text { hazardous purge water) }\end{array}$ & $\begin{array}{l}\text { Non-hazardous, contaminated waste } \\
\text { waters. Analytical results indicate } \\
\text { concentration in the water exceed the } \\
\text { MCL. These waters can contain nitrate } \\
\text { concentration }>100 \mathrm{mg} / \mathrm{L} \text {, Uranium } \\
>0.03 \mathrm{mg} / \mathrm{L} \text {, and Uranium isotopes }>4 \% \\
\text { of DCG. Waters commonly contain the } \\
\text { following typical halogenated } \\
\text { compounds (not inclusive) that exceed } \\
\text { the MCL, but are below RCRA TCLP } \\
\text { levels, include: Tetrachloroethene, } \\
\text { Trichloroethene, cis-1,2-Dichloroethene, } \\
\text { Carbon Tetrachloride, 1,1- } \\
\text { Dichloroethane, Methylene Chloride, } \\
\text { and Vinyl Chloride. Other } \\
\text { radioisotopes present consist of Tc-99 } \\
\text { and daughter products of Uranium. } \\
\text { Although not regulated, this waste water } \\
\text { is contained, handled, and sent for } \\
\text { disposal as a Best Management Practice } \\
\text { (BMP) at Y-12. As a BMP, this } \\
\text { contaminated purge water is not place on } \\
\text { clean surfaces (soils) or near surface } \\
\text { water tributaries. Annual groundwater } \\
\text { data evaluation, multiple sampling } \\
\text { event, and groundwater plume } \\
\text { characteristics provide ample evidence } \\
\text { of this classification. }\end{array}$ & $\begin{array}{l}\text { Segregate non-regulated waste } \\
\text { waters from other GWPP } \\
\text { waste waters that contain a } \\
\text { RCRA hazardous waste (SID } \\
2214 \text { and 2216). } \\
\text { Waste streams }{ }^{1} \text { have been } \\
\text { characterized and established } \\
\text { per well location and are } \\
\text { published in GWPP's annual } \\
\text { GWPP Sampling and Analysis } \\
\text { Plan. } \\
\text { Waters can be combined and } \\
\text { bulk as necessary in a DOT } \\
\text { approved container. }\end{array}$ & $\begin{array}{l}\text { Place in a DOT approved } \\
\text { container. } \\
\text { The above containers are } \\
\text { compatible with the } \\
\text { purge water and meet } \\
\text { packaging requirements } \\
\text { specified in Master } \\
\text { Profile WW-01 } \\
\text { Label containers in } \\
\text { accordance with Y71- } \\
\text { 310, Waste Container } \\
\text { Labeling }\end{array}$ & $\begin{array}{l}\text { SID } 2212 \text { waste stream meets the } \\
\text { waste acceptance criteria of Master } \\
\text { Profile WW-01. Sampling data is } \\
\text { used to complete Attachment G of } \\
\text { UCN } 2109 \text {. If Uranium is present, } \\
\text { above detection levels, then a wt } \\
\text { \%U235 sample is required to } \\
\text { determine enrichment, and a } \\
\text { duplicate sample is required if } \\
\text { results are >0.93 wt U235. All } \\
\text { other constituents listed in WW-01 } \\
\text { have been quantified through } \\
\text { current analytical results, previous } \\
\text { analyses, historical data (prior to } \\
\text { 1996), and groundwater plume } \\
\text { composition. A Process Knowledge } \\
\text { form attached to each UCN } 2109 \\
\text { documents the presence of } \\
\text { constituents seen in SID } 2212 \\
\text { waters and the absence of other such } \\
\text { constituents. This waste stream is } \\
\text { disposed at Y-12 National Security } \\
\text { Complex's onsite treatment facility } \\
\text { with authorization from Waste } \\
\text { Treatment Operations. Depending } \\
\text { on enrichment content, normal } \\
\text { disposal would be at either the West } \\
\text { End Treatment Facility (WETF) or } \\
\text { the Central Pollution Control } \\
\text { Facility (CPCF). }\end{array}$ \\
\hline
\end{tabular}




\begin{tabular}{|c|c|c|c|c|}
\hline Waste stream & Characterization $^{3}$ & Segregation Requirements & Packaging & Disposal Path \\
\hline $\begin{array}{l}\text { SID }^{1} 2214 \text { purge water } \\
\text { (purge water from } \\
\text { multiple F-listed RCRA } \\
\text { groundwater wells, along } \\
\text { with rinse waters from } \\
\text { sampling equipment and } \\
\text { disposables, bulked into } \\
\text { the same drum. All waste } \\
\text { water carries the F039 } \\
\text { waste code). }\end{array}$ & $\begin{array}{l}\text { Hazardous waste waters (no radiological } \\
\text { contaminants). Characterization based } \\
\text { upon well location. Wells located down- } \\
\text { gradient of the Bear Creek Burial } \\
\text { Grounds between north tributary (NT) } 6 \\
\text { and NT 8, and north of Bear Creek. } \\
\text { Purge water most likely contains } \\
\text { leachate from the BCBGs and is } \\
\text { considered RCRA F-listed (40 CFR Part } \\
\text { 261.31) based on established } \\
\text { documentation (F039 leachate is } \\
\text { comprised of F codes: F001, F002, } \\
\text { F004, and F005). } \\
\text { Typical halogenated volatile organic } \\
\text { compounds detected in the SID 2214 } \\
\text { waters, which are above the MCL } \\
\text { include: Tetrachloroethene, } \\
\text { Trichloroethene, 1,2-Dichloroethene, } \\
\text { 1,1-Dichloroethene, } \\
\text { 1,1,1-Trichloroethane, 1,1- } \\
\text { Dichloroethane, Methylene Chloride, } \\
\text { and Vinyl Chloride. Typically Benzene } \\
\text { and other total petroleum hydrocarbons } \\
\text { have also been identified. }\end{array}$ & $\begin{array}{l}\text { Segregate RCRA F-listed } \\
\text { waste waters from non- } \\
\text { regulated waste waters (SID } \\
\text { 2212) and RCRA } \\
\text { Characteristic waste waters } \\
\text { (SID 2216). } \\
\text { Waste waters are } \\
\text { bulked/accumulated at RCRA } \\
\text { Satellite Accumulation Area } \\
\text { (SAA) \#SA-993, under the } \\
\text { direction of the SAA Operator } \\
\text { or Alternate Operator }\end{array}$ & $\begin{array}{l}\text { Place in a DOT approved } \\
\text { container. } \\
\text { Waste is transported as } \\
\text { DOT Class } 9 \text { under a } \\
\text { Bill of Lading listing the } \\
\text { assigned EPA waste } \\
\text { code. Transporter has } \\
\text { received DOT training. } \\
\text { The above containers are } \\
\text { compatible with the } \\
\text { purge water and meet } \\
\text { packaging requirements } \\
\text { specified in Master } \\
\text { Profile WW-01 } \\
\text { Label containers in } \\
\text { accordance with Y71- } \\
310 \text {, Waste Container } \\
\text { Labeling }\end{array}$ & $\begin{array}{l}\text { Send SID } 2214 \text { waste waters to 90- } \\
\text { Day Yard for further management. } \\
\text { RCRA F-listed waste are } \\
\text { prohibited under Master Profile } \\
\text { WW-01, except under special } \\
\text { arrangement with DOE, or approved } \\
\text { by Waste Treatment Coordinator for } \\
\text { waste that can be treated at CPCF or } \\
\text { Groundwater Treatment Facility } \\
\text { (GWTF). SID } 2214 \text { waste waters } \\
\text { have been approved for treatment at } \\
\text { GWTF with the following } \\
\text { prohibitions: waters with Uranium } \\
\text { above detection (based on waste } \\
\text { sample analyses) and Nitrates in } \\
\text { concentration > 100 mg/L. All } \\
\text { other constituents listed in WW-01 } \\
\text { have been quantified through } \\
\text { current analytical results, previous } \\
\text { analyses, historical data (prior to } \\
\text { 1996), and groundwater plume } \\
\text { composition. A Process Knowledge } \\
\text { form attached to each UCN } 2109 \\
\text { documents the presence and absence } \\
\text { of WW-01 constituents seen in SID } \\
\text { 2214 waters. This waste stream is } \\
\text { disposed at Y-12 National Security } \\
\text { Complex's onsite treatment facility } \\
\text { with authorization from Waste } \\
\text { Treatment Operations. }\end{array}$ \\
\hline
\end{tabular}




\begin{tabular}{|c|c|c|c|c|}
\hline Waste stream & Characterization $^{3}$ & Segregation Requirements & Packaging & Disposal Path \\
\hline $\begin{array}{l}\text { SID }^{1} 2216 \text { purge water } \\
\text { (purge water from } \\
\text { multiple RCRA } \\
\text { characteristic wells } \\
\text { bulked into the same } \\
\text { drum. The EPA waste } \\
\text { code is dependent on the } \\
\text { well location). }\end{array}$ & $\begin{array}{l}\text { Hazardous waste waters (mixed and } \\
\text { non-radiological contaminated). } \\
\text { Analytical results indicate that } \\
\text { concentrations exceed a RCRA Toxicity } \\
\text { Contaminant Leaching Procedure (TCLP } \\
\text { - 40 CFR Part 261.24). Annual } \\
\text { groundwater data evaluation, plume } \\
\text { evaluations, and repeated sampling } \\
\text { events give weighted evidence to this } \\
\text { classification (wells may receive this } \\
\text { classification if concentrations have } \\
\text { been consistently approaching the } \\
\text { RCRA TCLP levels). } \\
\text { SID 2216 waste water can contain the } \\
\text { following EPA waste codes: } \\
\text { D005 - Barium } \\
\text { D006 - Cadmium } \\
\text { D018 - Benzene } \\
\text { D019 - Carbon Tetrachloride } \\
\text { D029 - 1,1-Dichloroethene } \\
\text { D039 - Tetrachloroethene } \\
\text { D040 - Trichloroethene } \\
\text { D043 - Vinyl Chloride } \\
\text { In addition to the above, these waters } \\
\text { may contain the following volatile } \\
\text { organic compounds: } 1,2-D i c h l o r o e t h e n e, \\
1,1,2-\text { Trichloro-1,2,2-triflouroethane, } \\
\text { 1,1,1-Trichloroethane, } \\
\text { Dichloroethane, Acetone, Methylene } \\
\text { Chloride, Chloroform and other total } \\
\text { petroleum hydrocarbons. These waters } \\
\text { may can contain trace metals, nitrate } \\
\text { concentration > 100 mg/L, Uranium } \\
>0.03 \text { mg/L, Uranium isotopes }>4 \% \text { of } \\
\text { DCG, and other radioisotopes (Tc99 and } \\
\text { daughter products of Uranium). }\end{array}$ & $\begin{array}{l}\text { Segregate RCRA } \\
\text { characteristic waste waters } \\
\text { from non-regulated waste } \\
\text { waters (SID 2212) and RCRA } \\
\text { F-listed waste waters (SID } \\
\text { 2216). } \\
\text { RCRA characteristic waste } \\
\text { waters are bulked/accumulated } \\
\text { at RCRA Satellite } \\
\text { Accumulation Area (SAA) } \\
\text { \#SA-992, under the direction } \\
\text { of the SAA Operator or } \\
\text { Alternate Operator }\end{array}$ & $\begin{array}{l}\text { Place in a DOT approved } \\
\text { container. } \\
\text { Waste is transported as } \\
\text { DOT Class } 9 \text { under a } \\
\text { Bill of Lading listing the } \\
\text { assigned EPA waste } \\
\text { code. Transporter has } \\
\text { received DOT training. } \\
\text { The above containers are } \\
\text { compatible with the } \\
\text { purge water and meet } \\
\text { packaging requirements } \\
\text { specified in Master } \\
\text { Profile WW-01 } \\
\text { Label containers in } \\
\text { accordance with Y71- } \\
\text { 310, Waste Container } \\
\text { Labeling }\end{array}$ & $\begin{array}{l}\text { Send SID } 2216 \text { waste waters to } 90- \\
\text { Day Yard for further management. } \\
\text { SID } 2216 \text { waste waters meet the } \\
\text { waste acceptance criteria of Master } \\
\text { Profile WW-01. Waters with } \\
\text { Uranium above detection (based on } \\
\text { waste sample analyses) require a wt } \\
\% \text { 235 sample to determine } \\
\text { enrichment and a duplicate sample } \\
\text { is required if results are }>0.93 \text { wt } \\
\text { U235. Nitrates concentration }>10 \\
\text { mg/L must be indicated. All other } \\
\text { constituents listed in WW-01 have } \\
\text { been quantified through current } \\
\text { analytical results, previous analyses, } \\
\text { historical data (prior to 1996), and } \\
\text { groundwater plume composition. A } \\
\text { Process Knowledge form attached to } \\
\text { each UCN } 2109 \text { documents the } \\
\text { presence and absence of WW-01 } \\
\text { constituents seen in SID } 2216 \\
\text { waters. This waste stream is } \\
\text { disposed at Y-12 National Security } \\
\text { Complex's onsite treatment facility } \\
\text { with authorization from Waste } \\
\text { Treatment Operations. Depending } \\
\text { on enrichment content, waste waters } \\
\text { are disposed at the West End } \\
\text { Treatment Facility (WETF) or the } \\
\text { Central Pollution Control Facility } \\
\text { (CPCF). }\end{array}$ \\
\hline
\end{tabular}




\begin{tabular}{|c|c|c|c|c|}
\hline Waste stream & Characterization $^{3}$ & Segregation Requirements & Packaging & Disposal Path \\
\hline $\begin{array}{l}\text { Disposables and sampling } \\
\text { equipment in contact } \\
\text { with RCRA } \\
\text { characteristic or F-listed } \\
\text { purge water: } \\
\text { Sampling equipment: } \\
\text { includes sample pumps, } \\
\text { tubing, sample trays, and } \\
\text { flow-through cells (all } \\
\text { components). These items } \\
\text { meet the definition of a } \\
\text { "container” under RCRA. } \\
\text { Non-absorbent } \\
\text { disposables - include: } \\
\text { gloves, plastic bags, and } \\
\text { instrument probes } \\
\text { Absorbent disposables - } \\
\text { includes paper towels, } \\
\text { wipes, clothes, litmus } \\
\text { paper. During normal } \\
\text { sampling operations these } \\
\text { items should not come } \\
\text { into contact with RCRA } \\
\text { characteristic or F-listed } \\
\text { waste waters. }\end{array}$ & $\begin{array}{l}\text { Non-hazardous solid waste and RCRA } \\
\text { empty containers. The sampling } \\
\text { equipment and disposables which comes } \\
\text { in contact with RCRA purge waters will } \\
\text { not be subject to RCRA if: } \\
\text { 1) The waste can be sufficiently } \\
\text { removed from non-absorbent material } \\
\text { (disposables), such as nitrile gloves, } \\
\text { plastic surfaces, instrument probes, and } \\
\text { external surfaces of sample bottles by } \\
\text { rinsing such items. All rinse water must } \\
\text { be collected and bulked under the } \\
\text { appropriate RCRA waste stream (SID } \\
\text { 2216 or } 2214 \text { ). } \\
\text { 2) Sampling equipment that meets the } \\
\text { definition of a "container" under RCRA } \\
\text { and is not subject to regulation once } \\
\text { the container is “empty" as defined } \\
\text { under } \mathbf{4 0} \text { CFR Part 261.7, paragraph } \\
\text { (b). To meet this requirement all fluids } \\
\text { must be sufficiently drained from the } \\
\text { equipment, by normal means as possible, } \\
\text { and then rinsed at least once to remove } \\
\text { residue. All rinse water must be } \\
\text { collected and bulked under the } \\
\text { appropriate RCRA waste stream (SID } \\
\text { 2216 or } 2214 \text { ). } \\
\text { 3) Absorbent disposable such as wipes, } \\
\text { paper towels, or clothes that are use to } \\
\text { remove/clean/dry any addition } \\
\text { liquids/residues RCRA empty } \\
\text { containers, once the items are rinsed, are } \\
\text { also not subject to RCRA. Litmus } \\
\text { paper, if used for its intended purpose, } \\
\text { and does not come into contact with } \\
\text { F039 waste water, is also not subject to } \\
\text { RCRA. }\end{array}$ & $\begin{array}{l}\text { Segregate non-regulated } \\
\text { waste streams from those } \\
\text { items subject to RCRA. all } \\
\text { Sampling equipment: can be } \\
\text { reused as necessary for the } \\
\text { multiple sampling events and } \\
\text { are not regulated. } \\
\text { Non-absorbent disposables - } \\
\text { once rinsed are not regulated. } \\
\text { Absorbent disposables -. } \\
\text { Items used to wipe/dry/clean } \\
\text { RCRA empty containers are } \\
\text { not regulated and can also be } \\
\text { disposed of into sanitary trash } \\
\text { (profile S-020). If these items } \\
\text { do come into contact with } \\
\text { RCRA waste water, the items } \\
\text { are subject to regulation. } \\
\text { These items must be wrung } \\
\text { out as much as possible (water } \\
\text { collected) and segregated from } \\
\text { non-regulated items. }\end{array}$ & $\begin{array}{l}\text { ALL non-regulated } \\
\text { items - dispose of into } \\
\text { the appropriate sanitary } \\
\text { waste receptacle or } \\
\text { dumpster, as specified } \\
\text { under Master Profile S- } \\
020 \text {. } \\
\text { Any items subject to } \\
\text { RCRA regulation must } \\
\text { be place in a DOT } \\
\text { approved container and } \\
\text { labeled in accordance } \\
\text { with procedure Y71-310, } \\
\text { Waste Container } \\
\text { Labeling. Waste is } \\
\text { transported as DOT } \\
\text { Class } 9 \text { under a Bill of } \\
\text { Lading, listing the } \\
\text { assigned EPA waste } \\
\text { code, to the 90-Day Yard } \\
\text { for further management. } \\
\text { Transporter has received } \\
\text { DOT training. }\end{array}$ & $\begin{array}{l}\text { All the items listed below require } \\
\text { authorization from Y-12 Waste } \\
\text { Management prior to disposal in } \\
\text { Sanitary Trash } \\
\text { Sampling equipment - once the } \\
\text { item is no longer of use, or can no } \\
\text { longer be used, the item can be } \\
\text { disposed of in sanitary trash (Waste } \\
\text { Profile No. S-020). } \\
\text { Non-absorbent disposables - after } \\
\text { items are rinsed, collect the rinse } \\
\text { solution and bulk with SID } 2216 \text { or } \\
\text { 2214 purge water, and then dispose } \\
\text { of the item in sanitary trash (S-020). } \\
\text { Absorbent disposables - not } \\
\text { subject to regulation can be } \\
\text { disposed into sanitary trash (S-020). } \\
\text { Absorbent material that comes } \\
\text { into contact with } \\
\text { Characteristic (SID 2216) purge } \\
\text { water, by process knowledge the } \\
\text { whole material if tested under the } \\
\text { TCLP would not exceed TCLP } \\
\text { levels, and therefore the item can be } \\
\text { disposed into sanitary trash (S-020). } \\
\text { Absorbent material that comes } \\
\text { into contact with RCRA F-listed } \\
\text { waste waters (SID 2214) will be } \\
\text { subject to regulation and must be } \\
\text { send to the } 90-D a y \text { Yard for further } \\
\text { management (Master Profile HW- } \\
\text { 01). Final disposal path will be } \\
\text { determined by Navarro-GEM to an } \\
\text { off-site RCRA TSD. }\end{array}$ \\
\hline
\end{tabular}




\section{Waste Management Plan for Y-12 Groundwater Protection Program Sampling Activities}

\begin{tabular}{|c|c|c|c|c|}
\hline Waste stream & Characterization $^{3}$ & Segregation Requirements & Packaging & Disposal Path \\
\hline $\begin{array}{l}\text { All disposables } \\
\text { equipment used for } \\
\text { GWPP purposes (non F- } \\
\text { listed wells): } \\
\text { Sampling pumps, gloves, } \\
\text { wipes, tubing, litmus } \\
\text { paper, instrument probes, } \\
\text { sample trays, and flow- } \\
\text { through cells. }\end{array}$ & $\begin{array}{ll}\text { Non-hazardous solid } & \text { waste. } \\
\text { Characterization is not required. } & \end{array}$ & $\begin{array}{l}\text { Segregate F-listed } \\
\text { contaminated items from non } \\
\text { F-listed contaminated items. }\end{array}$ & N/A & $\begin{array}{l}\text { Items not in contact with any F- } \\
\text { listed purge water can be disposed } \\
\text { in sanitary trash (profile S020) with } \\
\text { authorization from Y-12 Waste } \\
\text { Management. } \\
\text { All sampling equipment is to be } \\
\text { reused till the item is no longer of } \\
\text { use and then disposed of in sanitary } \\
\text { trash. } \\
\text { All Sanitary waste placed in the } \\
\text { approved on-site Solid Waste } \\
\text { Disposal Facility (Industrial } \\
\text { Landfill) }\end{array}$ \\
\hline $\begin{array}{l}\text { Waters/Fluids generated } \\
\text { during well development } \\
\text { of existing wells (well } \\
\text { development is } \\
\text { performed on an as } \\
\text { needed basis, prior to } \\
\text { sampling, to maintain } \\
\text { groundwater flow to well. } \\
\text { Five to } 10 \text { well casing } \\
\text { volumes are generated) }\end{array}$ & $\begin{array}{l}\text { Well development of existing wells will } \\
\text { utilize the most recent sampling } \\
\text { analytical results and will follow the } \\
\text { three waste streams (SIDs) for purge } \\
\text { water. }\end{array}$ & $\begin{array}{l}\text { Segregate water based on the } \\
\text { three existing waste streams } \\
\text { for purge water }\end{array}$ & $\begin{array}{l}\text { Based on volume and } \\
\text { waste stream ID number. } \\
\text { Containers could consist } \\
\text { of drums, polytanks, or } \\
\text { tankers. }\end{array}$ & $\begin{array}{l}\text { See the three purge water waste } \\
\text { streams IDs above }\end{array}$ \\
\hline
\end{tabular}

1 “SID” - “Stream Identification Number” are the pre-established waste streams identification (ID) for purge waters generated at Y-12. These waste streams were established by Y-12 Waste Operations, prior to 1995, and have been utilized to segregate waste waters. The waste stream ID is established for the coming Calendar Year (CY) for each well location to be monitored; based upon characterization of the most recent sampling results for that well location. These are published an appendix to GWPP's annual Sampling and Analysis Plan (published 2-3 months prior the start of the CY), and the waste stream is established for any other wells added during that CY and documented in addenda to this plan.

"Purge Water" - unusable portion of groundwater purged from a well prior to sample collection. Water is in a liquid form,(99.9\% liquid) with normally < 100 mg/L of suspended solids. Water contains contaminants that are in solution (dissolved phase) with little sediment load.

${ }^{3}$ Analytical results (past and present) from sampling events are used to characterize purge water. The GWPP uses a standardized parameter list for every sample, which includes:

1. ICP metals (SW846- EPA 6010B), ICPMS metals (EPA-200.8), Mercury (SW846 - 7470) - includes Uranium metal (0.0005 mg/L)

2. Anions - Alkalinity, Chloride, Fluoride, Nitrates, Sulfates

3. Volatile Organic Compound - SW846 - EPA 8260B

4. Gross Alpha and Gross Beta (EPA-900.0)

The following radioisotopes have been analyzed for on an as needed basis: Tritium, Tc-99, Isotopic Uranium, Total Uranium and wt\% U235, and other heavy radioisotopes (Am241, Np 237, I129, Thorium, Radium). 


\section{Y-12 GWPP PURGE WATER MANAGEMENT}

Example of Waste Identification Tag (UCN 2114B)

for SID 2212 purge water

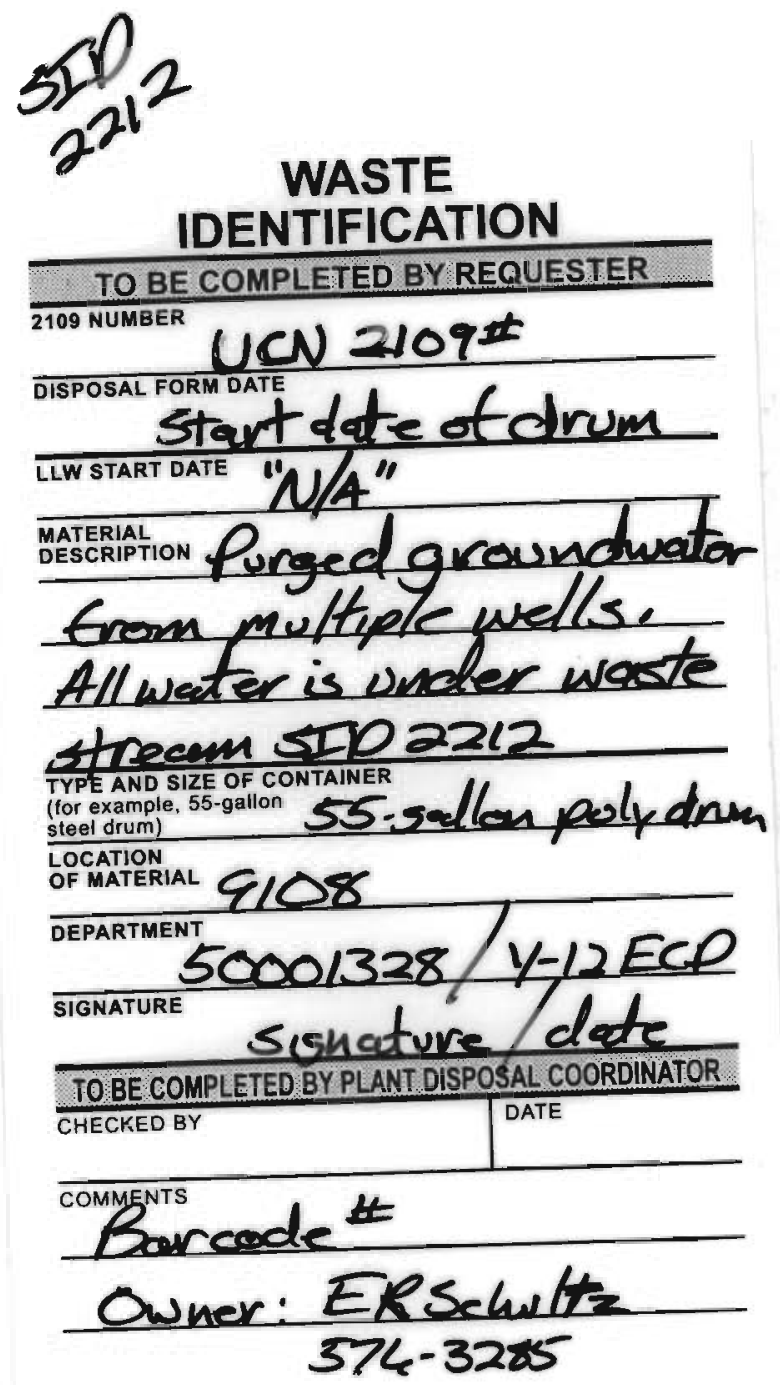

$\overline{\mathrm{UCN}-2114 \mathrm{~B}(11-05)}$ 


\section{Y-12 GWPP PURGE WATER MANAGEMENT}

Example of Hazardous Waste Identification Tag (UCN 2114A) for SID 2214 purge water

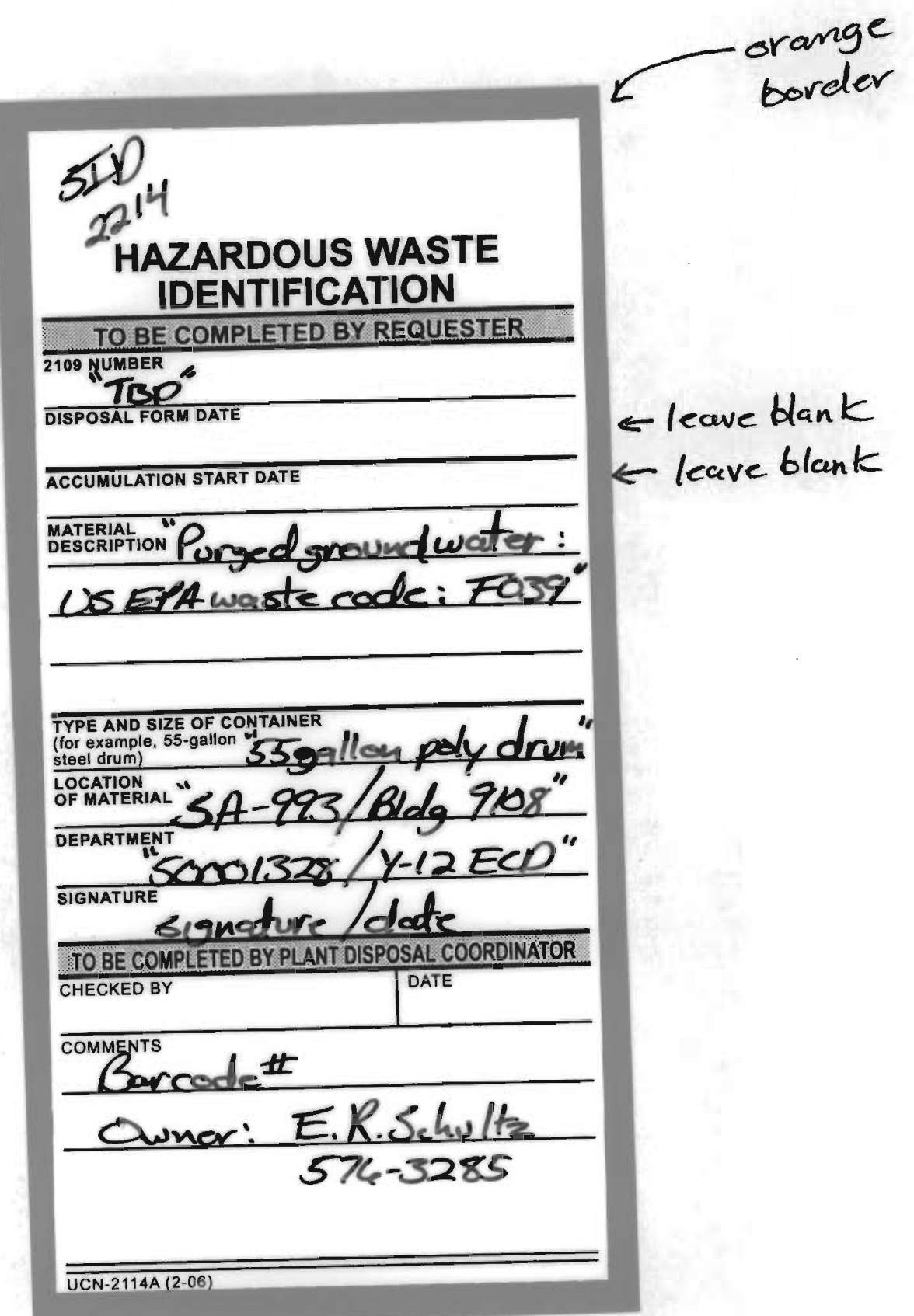


Example of Hazardous Waste Identification Tag (UCN 2114A) for SID 2216 purge water

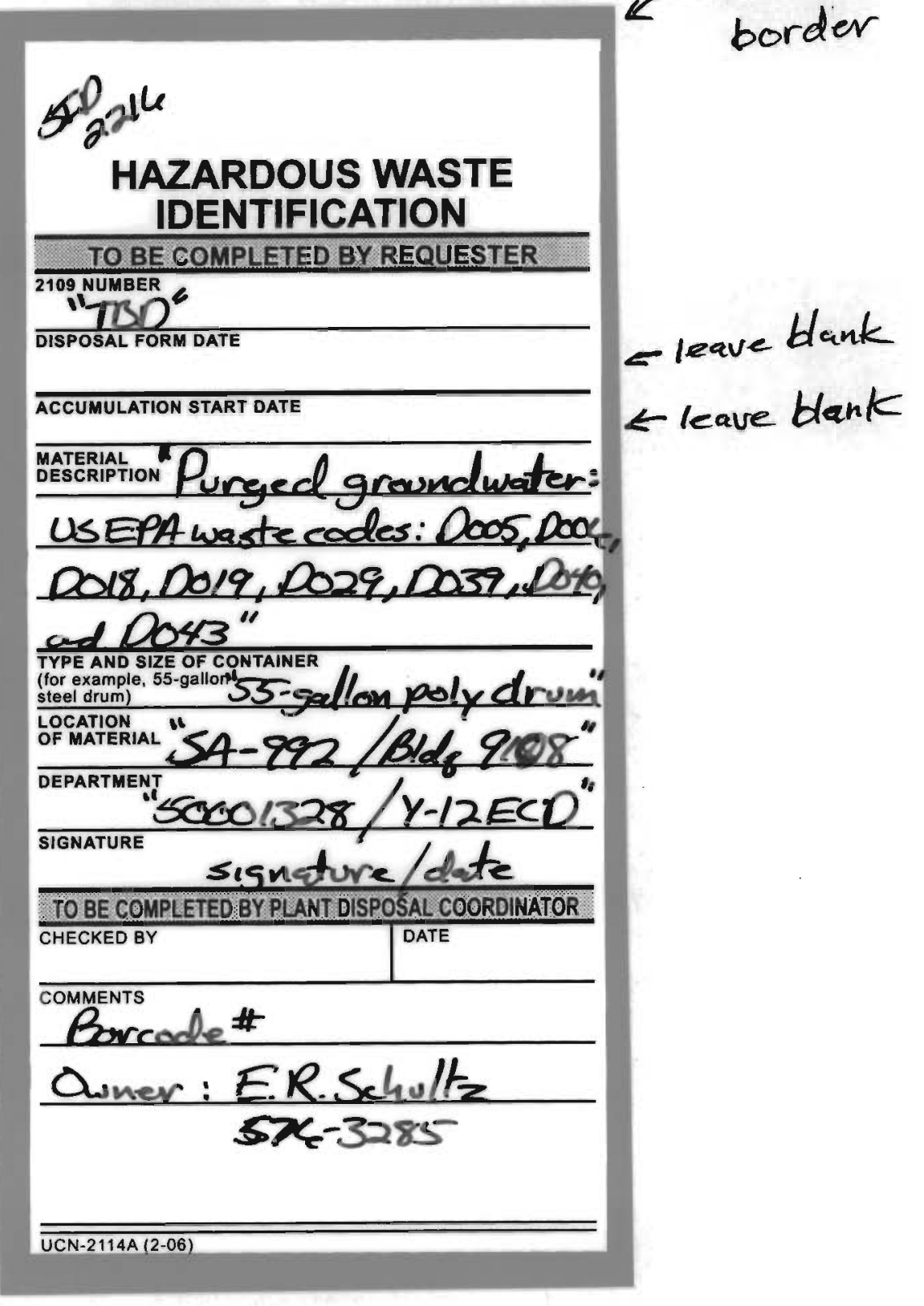


APPENDIX G.2

WASTE STREAM IDENTIFICATION FOR PURGED GROUNDWATER 
Table G.2. Waste stream identity and RCRA waste code for groundwater purged from wells to be sampled during CY 2008

\begin{tabular}{|c|c|c|c|c|c|c|c|}
\hline \multirow{2}{*}{ Regime } & \multirow{2}{*}{$\begin{array}{c}\text { CY } 2008 \\
\text { Locations }\end{array}$} & \multicolumn{4}{|c|}{ Quarter Sampled } & \multirow{2}{*}{$\begin{array}{c}\text { Waste Stream ID } \\
(\text { SID \#) }\end{array}$} & \multirow{2}{*}{ RCRA Waste Code } \\
\hline & & Q1 & $\mathbf{Q 2}$ & Q3 & Q4 & & \\
\hline \multirow[t]{45}{*}{ Bear Creek } & GW-006 & $\mathrm{Y}$ & & $\mathrm{Y}$ & & SID 2212 & \\
\hline & GW-014 & & & Y' & & SID 2214 & F039 \\
\hline & GW-058 & Y... & & & & Not Contained & $\cdot-11-1=1$ \\
\hline & GW-065 & Y... & & $\mathrm{Y}$ & & SID 2212 & \\
\hline & GW-068 & & & Y & & SID 2216 & D043 \\
\hline & GW-071 & $\mathrm{Y}$ & & Y & & SID 2214 & F039 \\
\hline & GW-082 & & & Y & & SID 2214 & F039 \\
\hline & GW-085 & 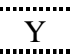 & & Y & & SID 2212 & \\
\hline & GW-089" & & & Y & & SID 2214 & F039 \\
\hline & GW-098 & Y & & & & SID 2212 & 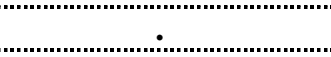 \\
\hline & GW-122 & Y.... & & & & SID 2212 & . \\
\hline & GW-225 & Y... & & $\mathrm{Y}$ & & SID 2212 & \\
\hline & GW-226 & Y & & Y & & SID 2212 & \\
\hline & GW-227 & Y & & & & SID 2212 & ......... \\
\hline & GW-229 & $Y$ & & & & SID 2212 & . \\
\hline & GW-246 & Y.... & & 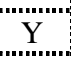 & & SID 2212 & . \\
\hline & GW-257 & & & Y & & SID 2214 & F039 \\
\hline & GW-259 & Y... & & Y & & SID 2212 & \\
\hline & GW-289 & & & Y... & & SID 2214 & F039 \\
\hline & GW-306 & Y & & Y & & SID 2212 & \\
\hline & GW-309 & Y & & Y & & SID 2212 & \\
\hline & GW-310 & Y & & & & SID 2212 & \\
\hline & GW-312 & Y & & & & SID 2212 & ................ \\
\hline & GW-314 & Y.... & & Y.. & & SID 2212 & \\
\hline & GW-315 & Y... & & & & SID 2212 & ................. \\
\hline & GW-365 & Y & & & & SID 2212 & \\
\hline & $\mathrm{GW}-367$ & 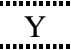 & & 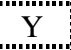 & & SID 2212 & ........... \\
\hline & GW-369 & 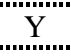 & & & & SID 2212 & (1) \\
\hline & $\mathrm{GW}-531$ & & & Y & & SID 2212 & - \\
\hline & $\mathrm{GW}-537$ & & & Y & & SID 2212 & \\
\hline & GW-615 & & & Y & & SID 2216 & D005 \\
\hline & GW-616 & & & Y & & SID 2212 & \\
\hline & GW-623 & $\mathrm{Y}$ & & Y & & SID 2214 & F039 \\
\hline & GW-626 & $\begin{array}{ll}3+\cdots \\
Y\end{array}$ & & Y..." & & SID 2214 & F039 \\
\hline & GW-627 & Y & & Y & & SID 2214 & F039 \\
\hline & GW-629 & Y & & Y & & SID 2214 & F039 \\
\hline & GW-648 & Y.." & & & & SID 2212 & \\
\hline & GW-653 & & & Y & & SID 2214 & F039 \\
\hline & GW-694 & $\begin{array}{ll}3.1 \\
Y\end{array}$ & & & & SID 2212 & \\
\hline & $\mathrm{GW}-703$ & Y... & & & & SID 2212 & \\
\hline & GW-724 & Y... & & & & SID 2212 & \\
\hline & GW-725 & Y... & & & & SID 2212 & \\
\hline & GW-738 & Y & & & & SID 2212 & \\
\hline & GW-740 & Y & & & & SID 2212 & .... \\
\hline & GW-829 & & & $\mathrm{Y}$ & & SID 2212 & ·."' \\
\hline
\end{tabular}


Table G.2 (continued)

\begin{tabular}{|c|c|c|c|c|c|c|c|}
\hline \multirow{2}{*}{ Regime } & \multirow{2}{*}{$\begin{array}{c}\text { CY } 2008 \\
\text { Locations }\end{array}$} & \multicolumn{4}{|c|}{ Quarter Sampled } & \multirow{2}{*}{$\begin{array}{c}\text { Waste Stream ID } \\
\text { (SID \#) }\end{array}$} & \multirow{2}{*}{ RCRA Waste Code } \\
\hline & & Q1 & Q2 & Q3 & Q4 & & \\
\hline \multirow[t]{8}{*}{ Chestnut Ridge } & GW-173 & & $\mathrm{Y}$ & & $\mathrm{Y}$ & SID 2212 & $\therefore$ \\
\hline & GW-175 & & & & Y & SID 2212 & $\therefore$ \\
\hline & GW-178 & & & & $\mathrm{Y}$ & Not Contained & \\
\hline & GW-322 & & & & Y'm & SID 2212 & - \\
\hline & GW-514 & & & & $\mathrm{Y}$ & Not Contained & \\
\hline & GW-608 & & & & Y & Not Contained & \\
\hline & GW-609 & & & & Y & Not Contained & \\
\hline & GW-612 & & & & Y & SID 2212 & ·. \\
\hline \multirow[t]{40}{*}{ East Fork } & $55-1 \mathrm{~A}$ & & Y & & & Not Contained & $\therefore$ \\
\hline & $55-2 \mathrm{~A}$ & & Y........ & & & SID 2212 & 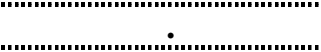 \\
\hline & $55-2 \mathrm{~B}$ & & Y & & & SID 2216 & D039 \\
\hline & $55-2 \mathrm{C}$ & & Y & & & SID 2216 & D039 \\
\hline & $55-3 A$ & & Y.." & & & SID 2216 & D039, D040 \\
\hline & $55-3 \mathrm{~B}$ & & Y & & & SID 2216 & D039, D040, D043 \\
\hline & $55-3 C$ & & Y & & & SID 2216 & D039, D040, D043 \\
\hline & $55-6 \mathrm{~A}$ & & Y.... & & & Not Contained & $\therefore$ \\
\hline & $56-1 \mathrm{~A}$ & & Y & & & Not Contained & $\cdot$ \\
\hline & $56-1 C$ & & Y & & & SID 2212 & $\therefore$ \\
\hline & $56-2 A$ & & Y & & & SID 2212 & ...... \\
\hline & $56-2 B$ & & Y & & & SID 2216 & D039 \\
\hline & $56-2 \mathrm{C}$ & & Y & & & SID 2216 & D039, D040 \\
\hline & 56-3A & & Y & & & SID 2212 & (1) \\
\hline & $56-3 B$ & & Y & & & SID 2212 & . \\
\hline & 56-3C & & Y... & & & SID 2216 & D039" \\
\hline & $56-4 \mathrm{~A}$ & & Y & & & SID 2212 & . \\
\hline & 56-6A & & Y & & & Not Contained & …‥ \\
\hline & $56-8 \mathrm{~A}$ & & Y & & & SID 2212 & $\therefore$ \\
\hline & $60-1 \mathrm{~A}$ & & ........ & & Y & Not Contained & $\therefore$ \\
\hline & GW-105 & & $\mathrm{Y}$ & & & SID 2212 & $\therefore$ \\
\hline & GW-106 & & Y & & & SID 2212 & $\cdot$ \\
\hline & GW-109 & & Y & & & SID 2216 & D006 \\
\hline & GW-148 & & & Y & & SID 2212 & $\therefore$ \\
\hline & GW-153 & & $\ldots$ & Y & & SID 2212 & $\therefore$ \\
\hline & GW-190 & & Y l & & & Not Contained & $\therefore$ \\
\hline & GW-192 & & Y & & & SID 2212 & (n)...... \\
\hline & $\mathrm{GW}-204$ & & & & Y & SID 2212 & . \\
\hline & $\mathrm{GW}-220$ & $\mathrm{Y}$ & & $\mathrm{Y}$ & & SID 2216 & D019 \\
\hline & GW-240 & & & Y & & SID 2212 & 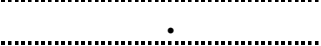 \\
\hline & GW-251 & & Y & & & SID 2212 & $\therefore$ \\
\hline & GW-265 & & $\mathrm{Y}$ & & & SID 2212 & $\cdot$ \\
\hline & GW-269 & & Y & & & SID 2212 & 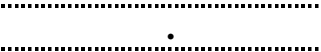 \\
\hline & GW-274 & & Y & & & SID 2216 & D039 \\
\hline & GW-275 & & $\mathrm{Y}$ & & & SID 2216 & D005 \\
\hline & GW-332 & & Y & & & SID 2216 & D039 \\
\hline & GW-336 & & 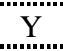 & & & SID 2212 & 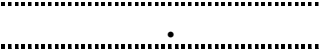 \\
\hline & GW-337 & & Y & & & SID 2216 & D039, D040 \\
\hline & $\mathrm{GW}-381$ & & & $\mathrm{Y}$ & & SID 2212 & - \\
\hline & "GW-383 & & & Y' & & "SID 2212 & '"' \\
\hline
\end{tabular}


Table G.2 (continued)

\begin{tabular}{|c|c|c|c|c|c|c|c|}
\hline \multirow{2}{*}{ Regime } & \multirow{2}{*}{$\begin{array}{c}\text { CY } 2008 \\
\text { Locations }\end{array}$} & \multicolumn{4}{|c|}{ Quarter Sampled } & \multirow{2}{*}{$\begin{array}{c}\text { Waste Stream ID } \\
\text { (SID \#) }\end{array}$} & \multirow{2}{*}{ RCRA Waste Code } \\
\hline & & Q1 & Q2 & Q3 & Q4 & & \\
\hline \multirow{31}{*}{$\begin{array}{c}\text { East Fork } \\
\text { (continued) }\end{array}$} & GW-508 & & $\mathrm{Y}$ & & $\mathrm{Y}$ & SID 2216 & D018 \\
\hline & GW-617 & & & & $\mathrm{Y}$ & Not Contained & 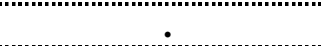 \\
\hline & GW-619 & & & & $Y$ & SID 2212 & $\therefore . .$. \\
\hline & GW-620' & & & & ' & "Not Contained & - \\
\hline & GW-633 & & Y & & & SID 2216 & D018 \\
\hline & GW-656 & & & & Y & SID 2216 & D040 \\
\hline & GW-686 & & & & Y & SID 2212 & 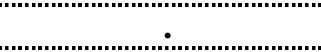 \\
\hline & GW-690 & & & & $\mathrm{Y}$ & SID 2212 & ...' \\
\hline & GW-691 & & & & $\mathrm{Y}$ & SID 2216 & D039 \\
\hline & GW-692 & & & & $\mathrm{Y}$ & SID 2212 & . \\
\hline & $\mathrm{GW}-698$ & & Y & & $\mathrm{Y}$ & SID 2216 & D040 \\
\hline & $\mathrm{GW}-700$ & & & & $\mathrm{Y}$ & SID 2212 & . \\
\hline & GW-722-14 & & & $\mathrm{Y}$ & & SID 2212 & ... \\
\hline & GW-722-17 & & & Y & & SID 2212 & $\cdot$ \\
\hline & GW-722-20 & & & Y..." & & SID 2212 & ........... \\
\hline & GW-722-22 & & & Y.." & & SID 2212 & • \\
\hline & GW-722-33 & & & Y & & Not Contained & ㄴ. \\
\hline & GW-744 & & & Y... & & Not Contained & ................ \\
\hline & $\mathrm{GW}-747$ & & & Y... & & Not Contained & \\
\hline & $\mathrm{GW}-748$ & & & $\mathrm{Y}$ & & Not Contained & \\
\hline & GW-763 & & & $\mathrm{Y}$ & & SID 2212 & $\therefore$ \\
\hline & GW-769 & & Y & & $\mathrm{Y}$ & SID 2212 & ...' \\
\hline & $\mathrm{GW}-770$ & & Y & & $\mathrm{Y}$ & SID 2212 & \\
\hline & GW-781 & & & & Y & Not Contained & $\therefore$ \\
\hline & GW-782 & & & & Y... & SID 2212 & $\therefore$ \\
\hline & $\mathrm{GW}-783$ & & & & $\mathrm{Y}$ & SID 2212 & ...... \\
\hline & GW-791 & & & & Y & SID 2212 & \\
\hline & GW-792 & & & & Y & "Not Contained & - \\
\hline & GW-816 & & & Y & & Not Contained & . \\
\hline & GW-820 & & Y.... & & 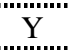 & SID 2216 & D039, D040 \\
\hline & GW-960 & & $\mathrm{Y}$ & & $\mathrm{Y}$ & Not Contained & $\cdot$ \\
\hline
\end{tabular}




\section{DISTRIBUTION}

\section{U.S. DEPARTMENT OF ENERGY}

J.P. Donnelly *

ENVIRONMENTAL COMPLIANCE DEPARTMENT

S.M. Field

C.C. Hill

S.B. Jones *

E.R. Schultz *

O.D. Stevens

L.O. Vaughan

GWPP-File *

ANALYTICAL CHEMISTRY ORGANIZATION

D.D. Altom

INFORMATION TECHNOLOGY

S.W. King

YDCC - RC *

Y-12 Central Files *

Y-12 Records Services (OSTI)

\section{ELVADO ENVIRONMENTAL LLC}

T.R. Harrison *

SCIENCE APPLICATIONS INTERNATIONAL CORPORATION

W.K. Jago

BECHTEL JACOBS COMPANY LLC

E.L. Berglund *

H.L. King

R.H. Ketelle *

L.M. Sims *

File-EMEF-DMC *

File-Y-12 Project PDCC *

COMMODORE ADVANCED SCIENCES, INC.

W. Foutz

UT-BATTELLE, LLC

D.B. Watson

TENNESSEE DEPARTMENT OF ENVIRONMENT AND CONSERVATION DOE OVERSIGHT

D. Gilmore *

Note: $*$ = receives hard copy version 